\title{
ANÁLISIS DE LOS EFECTOS DEL AUMENTO DEL SALARIO MÍNIMO SOBRE EL EMPLEO DE LA ECONOMÍA ESPAÑOLA
}

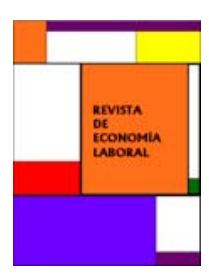

\author{
Inmaculada Cebrián*1, Joaquín Pitarch*, \\ César Rodríguez ${ }^{\dagger}$, Luis Toharia* \\ *Universidad de Alcalá de Henares, ${ }^{\dagger}$ Universidad de Oviedo
}

Recibido el 10 de julio de 2009; aceptado el 15 de enero de 2010

\section{Resumen}

El objetivo del artículo consiste en estimar el efecto de los aumentos en el salario minimo interprofesional sobre el empleo de los trabajadores españoles. Utilizando datos agregados de la Encuesta de Población Activa, se tratará de calcular la elasticidad de respuesta de la tasa de empleo con respecto a los cambios del indice de Kaitz para diversos colectivos. Además, también se tratará de analizar el efecto de los cambios en el índice de Kaitz sobre el nivel empleo de las empresas españolas a partir de los microdatos de la Encuesta de Coyuntura Laboral. Los resultados muestran la existencia de un efecto negativo muy pequeño del índice de Kaitz sobre los niveles de empleo de las empresas y de un efecto nulo sobre la tasa de empleo, incluso en el caso de los adolescentes.

Palabras clave: Salario Minimo, empleo, desempleo.

Clasificación JEL: J31, J23.

\section{Abstract}

The aim of the paper is to estimate the effects of the rise in the minimum wage on employment. By using aggregate data from the 'Encuesta de Población Activa' (EPA), we try to assess the elasticity of employment rate with respect to changes in the Kaitz Index (KI). Besides, we also try to analyze the effect of the changes in KI on the level of employment of Spanish firms, from the 'Encuesta de Coyuntura Laboral' (ECL) microdata. The outcomes show a small negative effect of KI over the level of employment of Spanish firms and a null effect over the employment rate, even in the case of teenagers.

Key words: Minimum wage, employment, unemployment.

JEL Classification: J31, J23.

1 Autora para correspondencia: inmaculada.cebrian@uah.es. Esta investigación ha sido financiada por el Ministerio de Trabajo e Inmigración. Su origen está en el Informe titulado Estudio de los efectos de un aumento del salario mínimo interprofesional en el empleo, realizado por los mismos autores.

(C) Revista de Economía Laboral 


\section{Introducción}

El Salario Mínimo Interprofesional (SMI) es un instrumento utilizado por los Gobiernos para tratar de resolver los problemas asociados a los empleos marginales muy poco cualificados, ocupados en muchos casos por adolescentes y mujeres. Se supone que sin la existencia de un SMI los ingresos laborales de estos trabajadores podrían ser tan reducidos que se mantendrían permanentemente en una situación de marginalidad (un circulo vicioso de baja cualificación-reducida motivación-pobreza-bajo salario). No obstante, el SMI puede tener también efectos negativos. Así, los autores que han analizado los efectos económicos del SMI han constatado en muchos países, incluido España, que las elevaciones del SMI pueden afectar negativamente al empleo precisamente en esos colectivos a los que el SMI pretende ayudar (adolescentes). Es decir, el SMI puede hacer que tengamos adolescentes mejor pagados pero una tasa de empleo para ese colectivo más reducida.

Desde el año 2004, el Gobierno sitúa entre sus prioridades la dignificación de la cuantía del salario mínimo, tratando de que éste recupere el poder adquisitivo perdido en los años anteriores sin perjudicar la creación de empleo. En concreto, el Gobierno de España pretende alcanzar un SMI de $800 €$ al mes (en 14 pagas anuales) en el año 2012 para tratar de lograr el objetivo fijado por la Carta Social Europea: que el SMI llegue a representar el 60 por ciento de los salarios medios. Teniendo en cuenta que en el año 2009 el SMI es de 624 euros mensuales, este objetivo conlleva un aumento del 28 por ciento a lo largo de los próximos 3 años, objetivo sin duda difícil de alcanzar dada la profundidad de la crisis que afecta al mercado de trabajo en nuestro país. Realmente, el objetivo del Gobierno se planteó en un contexto económico completamente diferente al actual (un mercado de trabajo con más de veinte millones de trabajadores y una tasa de paro alrededor del 8 por ciento), contexto en el que la subida del SMI no debería tener ninguna incidencia negativa sobre el empleo. Sin embargo, en la actualidad se corre el riesgo de que incrementos importantes del SMI añadan más problemas a la creación de empleo, e incluso al mantenimiento de los puestos de trabajo existentes.

En este contexto, el objetivo central de la presente investigación consiste en estimar el efecto de los aumentos en el SMI sobre el empleo de la economía española. Para realizar la investigación se utilizarán básicamente los datos procedentes de la Encuesta de Población Activa (EPA), la Encuesta Trimestral de Coste Laboral (ETCL), y la Encuesta de Coyuntura Laboral (ECL). 
El resto del artículo se estructura del siguiente modo. En la Sección 2, se expone el análisis teórico de los efectos del salario mínimo (SM) sobre el empleo. A continuación, en la Sección 3 se analiza la evolución del SMI en España. En la Sección 4 se estima un modelo para cuantificar la influencia de las variaciones del SMI sobre el nivel de empleo y sobre la tasa de empleo de la economía española. Por último, en la Sección 5 se resumen las principales conclusiones del estudio.

\section{Efectos del salario mínimo sobre el empleo.}

Los efectos teóricos de la existencia del SM sobre el empleo pueden analizarse en dos contextos distintos, dependiendo de si el mercado de trabajo es competitivo o no competitivo.

\subsection{El caso del mercado de trabajo competitivo}

En el marco de un mercado laboral perfectamente competitivo, el establecimiento por parte del Gobierno de un SM podría tener un efecto destructor del empleo sólo si el SM estuviera por encima del salario de equilibrio. En caso contrario, el SM resultaría absolutamente irrelevante. ${ }^{2}$ En el Gráfico 1 se muestran las dos posibilidades mencionadas. Cabe añadir que los efectos del SM sobre el empleo en un contexto competitivo dependen de su cuantía, pero también de la posición de la oferta y la demanda de trabajo; esto es, de la cualificación de los trabajadores y de la evolución del ciclo económico. Por ejemplo, si sube el SM y, simultáneamente, sube la demanda de trabajo (fase de auge) y mejora el nivel de cualificación de los trabajadores, los efectos negativos del SM serán despreciables. Por el contrario, si cae la demanda (fase de crisis) y crece la proporción de trabajo no cualificado en la economía, entonces la subida del SM podría ser lesiva para el empleo.

\subsection{El caso del mercado de trabajo no competitivo}

Los efectos teóricos de una subida del SM son muy diferentes en el caso de que el mercado de trabajo sea no competitivo. Por ejemplo, supongamos el caso de una empresa que no es salario-aceptante debido a que su conducta al contratar más o menos trabajo afecta al salario que

2 Un análisis más amplio de los efectos del salario mínimo en el marco del modelo competitivo puede consultarse en Brown, Gilroy y Cohen (1982). Estos autores extienden el análisis más simple a los casos de dos sectores (trabajadores cubiertos y no cubiertos), trabajo heterogéneo, retardos en los ajustes, y efectos sobre el bienestar. 
paga. Éste es el caso conocido como monopsonio. En esta situación, la empresa se enfrenta a una curva de oferta de trabajo de pendiente positiva (sólo puede extraer una unidad más de trabajo a cambio de pagar un salario más alto), siendo el Coste Marginal del Trabajo para la empresa superior al salario. En una situación de este tipo, la conducta maximizadora de la empresa al contratar trabajo consistirá en igualar el Coste Marginal del Trabajo al Ingreso del Producto Marginal. Como es bien conocido, en esta situación habrá un rango de variación del SM en el cual los incrementos en dicho salario conducirán a aumentos en el empleo, tal y como se recoge en el Gráfico 2. Partiendo de un salario inicial de monopsonio igual a Wm, la fijación de un Wmin1 por encima de Wm dará lugar a un aumento del empleo. Esto sucederá hasta que el salario alcance el valor competitivo, Wc. A partir de ahí los incrementos en el salario darán lugar a reducciones en el empleo (por ejemplo, para Wmin2).

\section{Gráfico 1 Efectos del salario mínimo en un mercado competitivo}

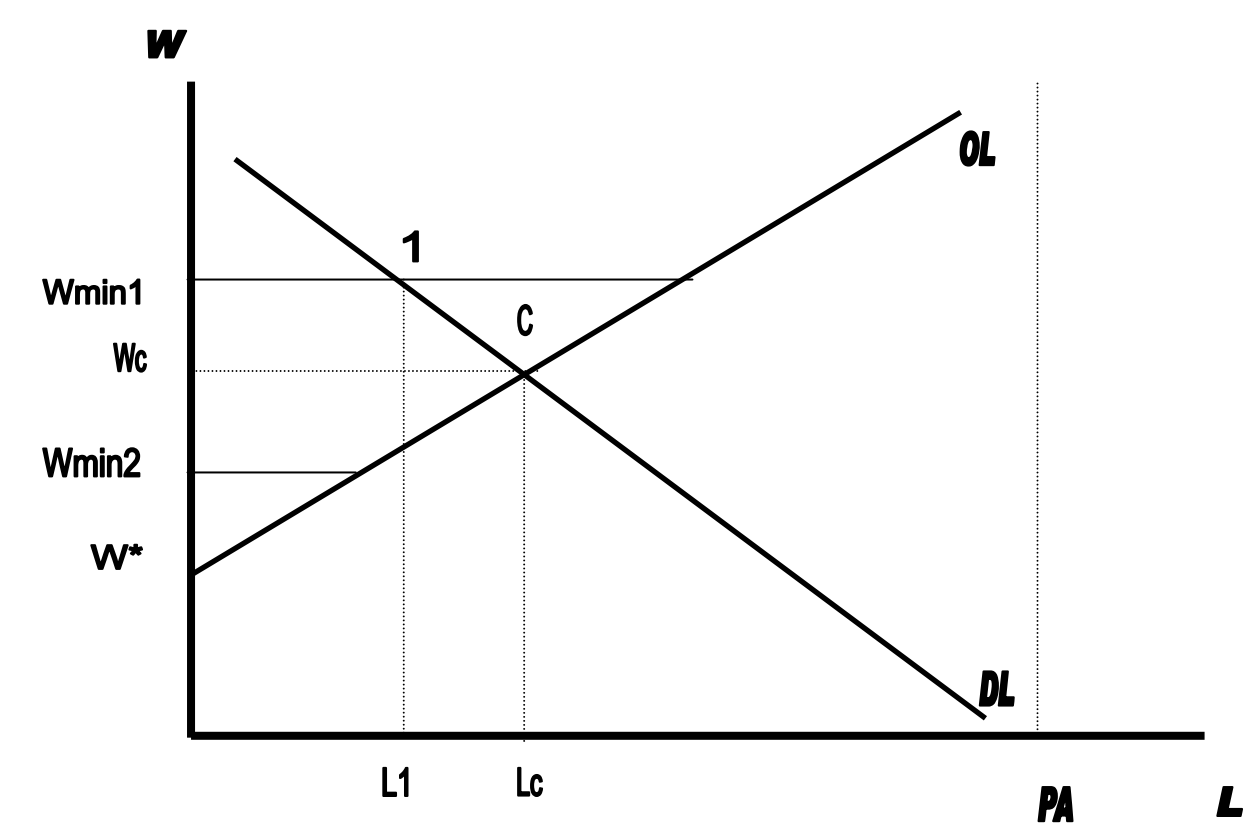

Obsérvese que la situación de monopsonio es un caso particular que corresponde a empresas con suficiente poder de mercado en la compra de factor trabajo como para poder situar los salarios por debajo del valor correspondiente a la competencia perfecta. Aunque este modelo no 
pretende describir el funcionamiento general del mercado de trabajo, como apuntan Dolado y Felgueroso (1997), los resultados del monopsonio pueden aparecer siempre que las empresas tengan algún poder a la hora de fijar los salarios de sus trabajadores. ${ }^{3}$

\section{Gráfico 2 Efectos del SM en un régimen de monopsonio}

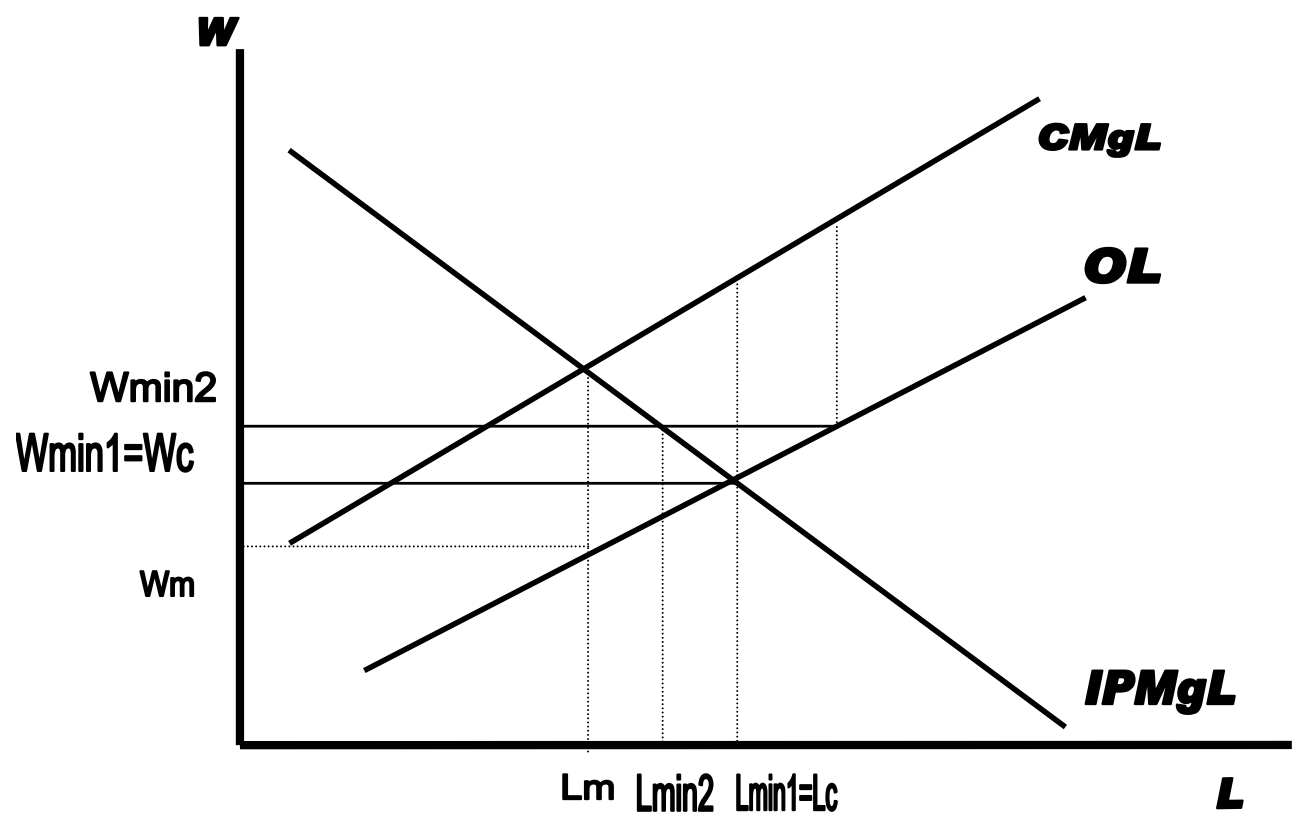

\subsection{El salario mínimo y los salarios de eficiencia}

La aproximación de los salarios de eficiencia contiene, a su vez, argumentos interesantes en favor de la elevación del SM bajo ciertas condiciones. Si se supone que el esfuerzo (productividad) de los trabajadores puede ser estimulado por la vía de los incrementos salariales, un aumento del SM podría elevar la productividad del trabajo y con ello la curva de demanda del factor, dando lugar a un aumento del empleo. En este contexto podrían ser compatibles las elevaciones simultáneas de

\footnotetext{
3 Para profundizar en el análisis teórico de los efectos del salario mínimo sobre el empleo en el marco del modelo de monopsonio y en otros ejemplos de competencia imperfecta, puede consultarse Manning (2003).
} 
salarios y empleo. ${ }^{4}$ A este argumento en favor de una elevación "prudente" del SM se pueden sumar otros. Por ejemplo, el aumento del SM permite a las familias dotarse a medio y largo plazo de un mejor nivel educativo y sanitario, lo que contribuye a una mejor disposición de la economía hacia el crecimiento. Este factor es especialmente relevante en los países que se encuentran en vías de desarrollo.

En definitiva, dado que el modelo competitivo es de difícil localización y el monopsonio puede existir sólo en algunos casos particulares, el análisis económico no es concluyente al prever los efectos del SM sobre el empleo. En este sentido, tal y como apunta Manning (2003), dada la dificultad de encontrar una respuesta concluyente en la teoría, la cuestión del impacto de los salarios mínimos sobre el empleo ha de resolverse fundamentalmente a partir de los estudios empíricos. ${ }^{5}$ En todo caso, parece claro que este efecto dependerá en gran medida de la evolución económica. Así, en un contexto de fuerte restricción en la demanda de trabajo (como el actual), en el que la oferta se caracteriza por la existencia de un amplio segmento de trabajo poco cualificado, sería posible que elevaciones fuertes del SMI dieran lugar a una reducción del empleo en el caso de los trabajadores menos cualificados.

\section{La evolución del SMI en España}

El SMI se define como la retribución mínima que ha de percibir un trabajador por su jornada legal de trabajo, con independencia del sector económico, tipo de contrato, nivel de cualificación, lugar de residencia y cualesquiera otras características personales (sexo, edad,...). Cada año, el Gobierno aprueba por medio de un Real Decreto las cuantías del SMI para el conjunto de los trabajadores y para dos grupos específicos: los eventuales y temporeros cuyos servicios a una misma empresa no excedan de 120 días, y el personal al servicio del hogar familiar que trabaje por horas. Desde el año 2004 el Gobierno está elevando el SMI por encima de la inflación prevista, al amparo del Real Decreto-Ley 3/2004, de 25 de junio, para la racionalización de la regulación del salario mínimo interprofesional y para el incremento de su cuantía. En ese Real Decretoley se crea una nueva figura, el Indicador Público de Renta de Efectos Múltiples (IPREM), que ha permitido desvincular al SMI de un variado

\footnotetext{
4 En este sentido, es interesante el trabajo de Georgiadis (2008), quien observa la existencia de un efecto positivo del salario mínimo sobre la productividad en el caso de un sector de bajos salarios de la economía británica.

5 Una revisión completa de los trabajos empíricos desarrollados desde comienzos de los 90 puede encontrarse en Neumark y Wascher (2007).
} 
conjunto de elementos de gasto público diferentes a los laborales, dando lugar así a crecimientos del mismo que en otro contexto serían muy difíciles de aplicar.

Otros rasgos importantes del SMI son los siguientes. El SMI se entiende fijado para la jornada legal en cada actividad, por lo que en el caso de jornadas inferiores el trabajador debe recibir la parte proporcional que supone dicha jornada. En segundo lugar, el SMI debe incrementarse en aquellos complementos salariales por rendimiento individual, por primas de producción y resultados de la empresa que se fijan en los convenios colectivos. Eso quiere decir que el SMI es la referencia fundamental para calcular los salarios base de convenio de las categorías profesionales inferiores. Como veremos más adelante, la negociación colectiva suele establecer salarios base superiores al SMI. Desde esta perspectiva, dado el elevado grado de cobertura de los convenios colectivos en nuestro país, el SMI se convierte en una garantía salarial únicamente para los pocos trabajadores que no están sujetos a un convenio colectivo.

\section{Gráfico 3 Evolución del SMI en términos nominales y reales. 1963- 2008 (Fuente: Boletín de Estadísticas Laborales (BEL)-MTIN e IPC-INE)}

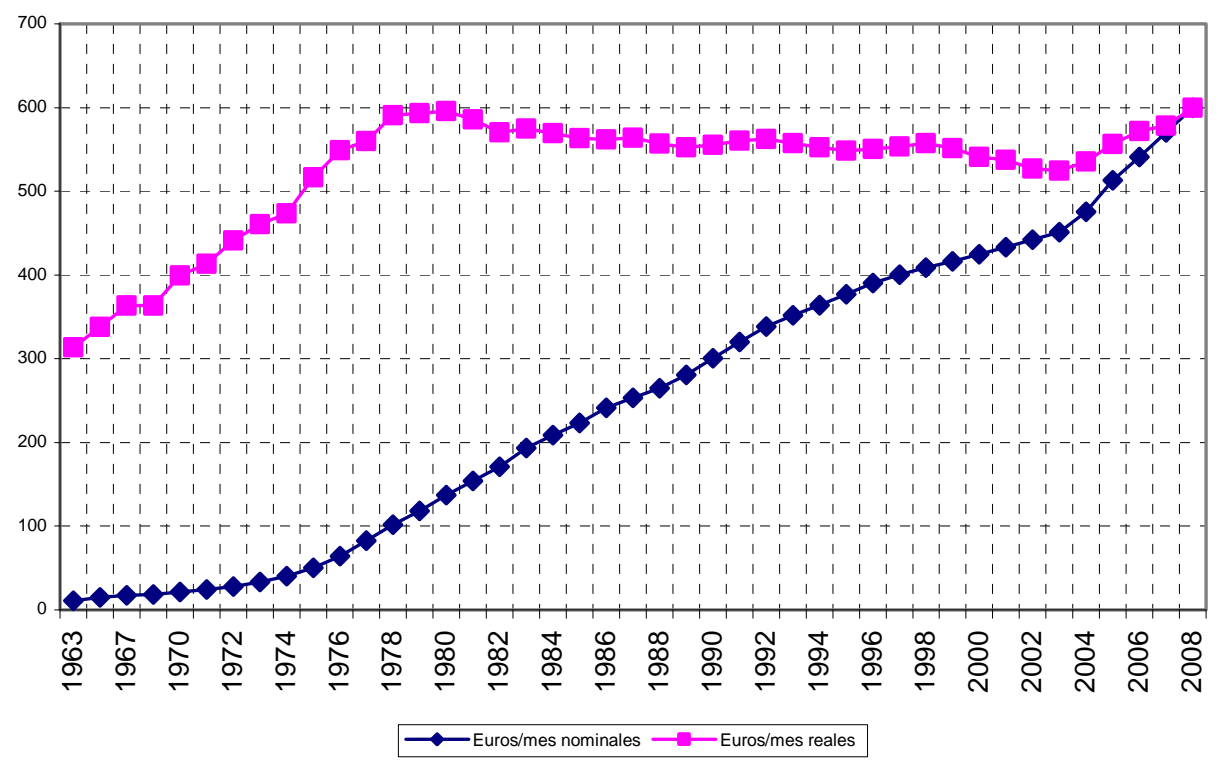

En la evolución del SMI se pueden observar tres periodos diferenciados. El primero, entre 1963 y 1989, en el que se distinguían tres 
Cebrián et al. / Revista de Economía Laboral 7 (2009), 1-38

tipos diferentes de SMI en función de la edad de los trabajadores. Los intervalos de edad fueron modificándose hasta establecerse en 1980 los siguientes: hasta 16 años, 17 años, y 18 o más años. El segundo periodo, desde 1990 hasta 1997, en el que se distinguían sólo dos colectivos (hasta 18 años y 18 y más); y el tercero, que arranca en 1998, en el que se fija un solo SMI.

Desde el año 1963, fecha de su implantación, el crecimiento del SMI medido en términos nominales no ha ido acompañado de un incremento de poder adquisitivo equivalente. Así, en el Gráfico 3 se observa que hasta el año 1980 el SMI real muestra una tendencia claramente creciente (de hecho el SMI se duplica en términos reales), pero a partir de este momento el SMI real inicia una suave caída que finaliza en el año 2003. En el año 2004 la evolución del SMI real se invierte gracias a la aprobación del Real Decreto-ley 3/2004, de 25 de junio. Obsérvese que en euros constantes (de diciembre de 2008), el SMI sólo logró recuperar el valor que tenía en 1980 en el año 2008, pese al importante incremento de productividad y nivel de vida experimentado en España desde esa fecha.

La medida que se utiliza en la práctica para conocer la importancia relativa del SMI es el índice de Kaitz (IK), que se define como el cociente entre el SMI y el salario medio de la economía. Existen varias opciones a la hora de elaborar este indicador relativo, dependiendo del concepto de salario medio utilizado, de si se toman salarios por hora, mes o año, y de si se emplean magnitudes brutas o netas (después de descontar impuestos y cotizaciones sociales).

En esta investigación, para representar el salario medio de la economía se empleará fundamentalmente el "coste salarial ordinario mensual por trabajador a tiempo completo" proporcionado por la Encuesta Trimestral de Coste Laboral (ETCL) del INE. Dado que la ETCL ofrece información sólo a partir del primer trimestre del año 2004, la construcción de una serie trimestral suficientemente larga nos lleva a emplear las series enlazadas de la Encuesta de Salarios en la Industria y los Servicios (ESIS; periodo 1981-2000) y del Índice de Costes Laborales (ICL; periodo 2001-2003). Por consiguiente, en principio es posible elaborar una serie trimestral del índice de Kaitz desde el primer trimestre de 1981 hasta la actualidad. No obstante, sólo es posible disponer de información sobre el coste salarial ordinario para los trabajadores contratados a tiempo completo desde el primer trimestre de 1996 en adelante. Debe tenerse en cuenta que el SMI es la referencia mínima de salario mensual para una jornada laboral a tiempo completo, por lo que el salario medio de comparación al elaborar el índice de Kaitz en términos de salario/mes también debe referirse a este tipo de dedicación laboral. Para el periodo 1981-1995 el coste salarial ordinario mensual que proporcionan 
las fuentes señaladas se refiere al total de los trabajadores (sin distinguir las dedicaciones a tiempo completo y tiempo parcial). Por ello, para construir una serie del índice de Kaitz a lo largo de este periodo, lo más correcto es utilizar magnitudes/hora. Esto es, habría que dividir el SMI por hora entre el coste salarial ordinario por hora efectivamente trabajada. Esta opción será la que se adopte, por ejemplo, en las estimaciones realizadas en la Sección 4.2 .

Volviendo al concepto de coste salarial ordinario mensual por trabajador a tiempo completo, esta magnitud incluye tanto el salario base como los complementos de periodicidad mensual, pero no las pagas extraordinarias. Por ello, el coste salarial "ordinario" es un buen elemento de referencia para la comparación del SMI mensual. ${ }^{6}$

Por otra parte, aunque el salario mínimo se define en términos brutos (antes de impuestos), es probable que para una buena parte de los trabajadores sujetos a dicho salario el mecanismo de deducciones en el impuesto sobre la renta de las personas físicas (IRPF) convierta esa cantidad en un ingreso neto (esto es, muchos de estos trabajadores pueden conseguir la devolución de las cantidades retenidas). Por este motivo, para elaborar el índice de Kaitz, el SMI mensual (que se supone finalmente neto) se compara con el coste salarial ordinario por trabajador y mes definido en términos netos. ${ }^{7}$ De lo contrario, el índice de Kaitz estaría infravalorando la proporción que representa el SMI en relación al salario medio de la economía. Es decir, podría ocurrir que un índice de Kaitz del 50 por ciento calculado con los datos brutos (coste salarial ordinario bruto en el denominador) fuese compatible con el objetivo del 60 por ciento en términos netos que recomienda la Carta Social Europea. Además, a las cantidades brutas (tanto al SMI como al coste salarial ordinario) se les quita también el importe de la cuota del trabajador a la Seguridad Social, al objeto de conocer la cuantía efectivamente percibida por el trabajador. Hay que tener en cuenta que para determinar el coste salarial ordinario ya se han excluido previamente las cotizaciones del empresario a la Seguridad Social.

Dada la información proporcionada por la ETCL, es posible calcular el índice de Kaitz para doce ramas de actividad, que son: industria extractiva, industria manufacturera, producción de energía

\footnotetext{
${ }^{6}$ Otra posible opción sería comparar el SMI anual (definido para catorce pagas) con el coste salarial total (que incluye los costes salariales ordinarios, pagos por horas extraordinarias, pagos extraordinarios y pagos atrasados). No obstante, apenas hay diferencias en los valores del índice de Kaitz calculados por ambos procedimientos.

7 Para calcular el coste laboral ordinario en términos netos, en esta investigación se utiliza la información proporcionada por la Agencia Tributaria sobre el tipo medio de retención por IRPF.
} 
eléctrica, construcción, comercio, hostelería, transporte, instituciones financieras, actividades inmobiliarias, educación, sanidad y otros servicios. ${ }^{8}$

\section{Gráfico 4 Índice de Kaitz (SMI mensual neto/Coste salarial ordinario mensual neto para los trabajadores a tiempo completo). 2000TI-2008TIV (Fuente: ETCL-INE y BEL-MTIN)}

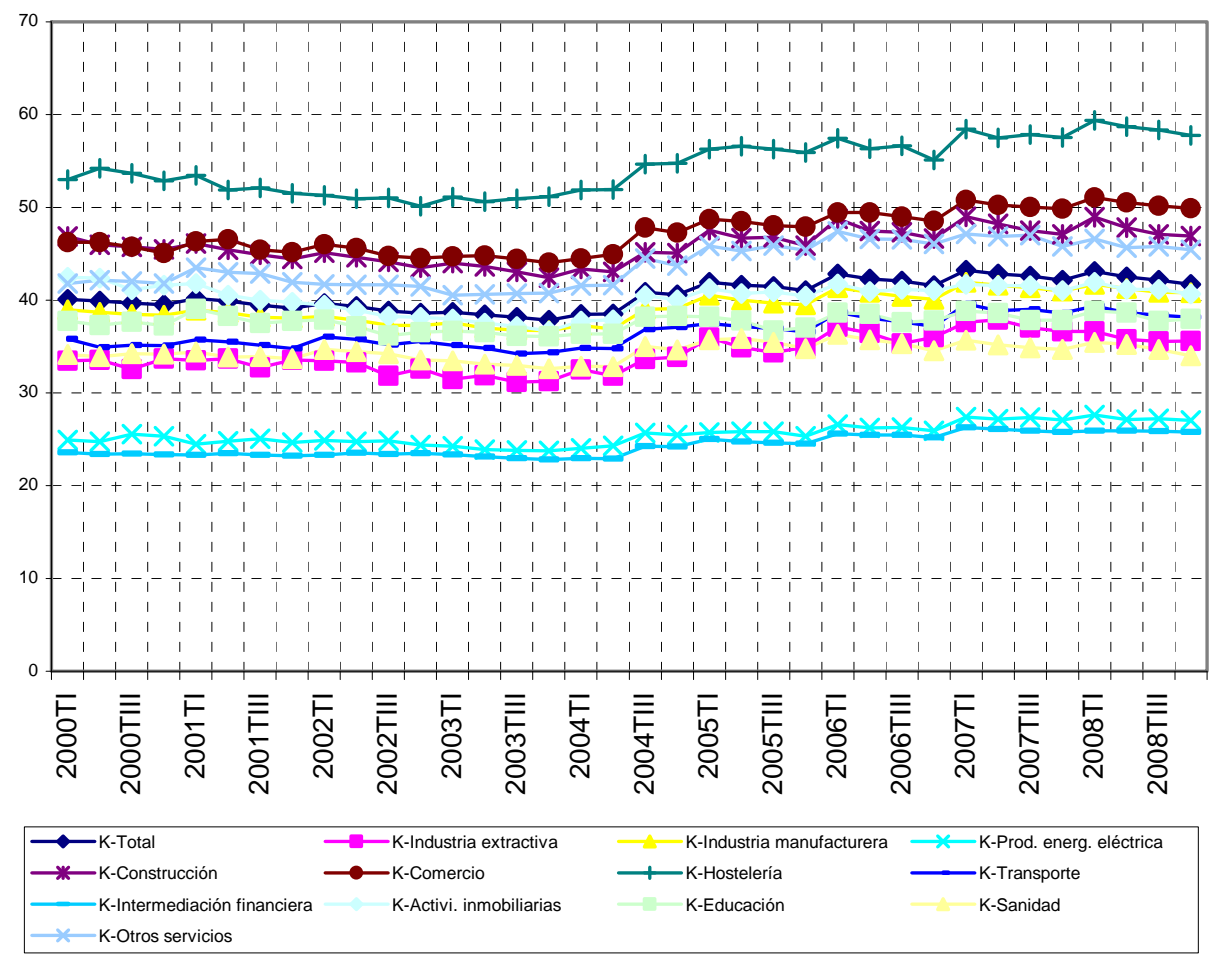

Por último, ya se ha señalado que en la evolución del SMI se pueden distinguir hasta 1997 varios periodos en función de los tramos de edad de los colectivos afectados por dicho salario. Este hecho hace que al elaborar el índice de Kaitz debamos definir un SMI ponderado, en el que el valor del SMI correspondiente a cada tramo de edad reciba un peso igual

\footnotetext{
${ }^{8}$ Hemos de destacar que la ETCL no incluye datos para el sector de la agricultura. Por ello, es posible que el verdadero índice de Kaitz para el conjunto de la economía española sea algo superior al valor que se presenta en este estudio, pues cabría esperar que la inclusión de la agricultura disminuyera algo el salario medio de la economía (que constituye el denominador del índice de Kaitz).
} 
al porcentaje que representan los ocupados en dicho tramo de edad sobre la cifra total de ocupados. ${ }^{9}$

Como ilustración de los valores que adopta el índice de Kaitz, el Gráfico 4 recoge su evolución entre los años 2000 y 2008 para las doce ramas de actividad anteriormente mencionadas. Obsérvese que en promedio para los cuatro trimestres de 2008 el SMI representaba algo más del 42 por ciento del salario medio de la economía española, habiéndose incrementado esta cifra en más de tres puntos desde el primer trimestre del año 2004 al cuarto trimestre de 2008. Por consiguiente, en el caso español el SMI está aún lejos de alcanzar el objetivo del 60 por ciento que recoge la Carta Social Europea.

Por otro lado, hemos de señalar que la negociación colectiva constituye en nuestro país un segundo escalón de salarios mínimos. En una investigación complementaria a ésta en la que se utilizan datos de convenios colectivos, se ha podido comprobar que los colectivos que podrían verse afectados por el SMI (jóvenes de categorías profesionales menos cualificadas), tienen en realidad unos salarios pactados superiores. ${ }^{10}$ Sólo en unos pocos de estos casos (trabajadores de menor edad y con contratos en prácticas o formación), se encuentran ejemplos de salarios negociados en convenio próximos al SMI. Como conclusión, es posible afirmar que el proceso de negociación colectiva origina en la práctica una densa red de salarios mínimos superpuestos que dejan en un papel secundario al propio SMI.

Finalmente, en cuanto a la incidencia del SMI, los datos procedentes de la Encuesta de Coyuntura Laboral (ECL) y de la Muestra Continua de Vidas Laborales (MCVL), ambas elaboradas por el Ministerio de Trabajo e Inmigración, indican que la cobertura o incidencia del SMI en nuestro país es muy reducida. En concreto, la ECL ofrecía para el año 1999 una tasa de incidencia del 2,5 por ciento, que se reducía a tan solo el 0,9 por ciento en el año 2008. Por su parte, según la MCVL, que aporta información sobre los episodios de empleo registrados en la Seguridad Social, el porcentaje de personas que se encontraban ocupadas en un empleo a tiempo completo y percibían una remuneración conforme a una base de cotización equivalente al SMI en el año 2004 casi alcanzaba al 1,5

\footnotetext{
9 Así, teniendo en cuenta el periodo de análisis elegido en la presente investigación, entre 1981 y 1989 el SMI de la economía se calcularía efectuando tres ponderaciones (utilizando los pesos de los ocupados de 16, 17 y 18 y más años en relación al total de ocupados); entre 1990 y 1997 se calcularía efectuando dos ponderaciones (menos de 18 y 18 y más años); y finalmente, a partir de 1998 el SMI a utilizar coincidiría con el vigente para todas las edades.

${ }^{10}$ En el trabajo de Cebrián et al. (2008) se puede encontrar un análisis de la relación del SMI con los salarios mínimos garantizados en una muestra de convenios colectivos.
} 
Cebrián et al. / Revista de Economía Laboral 7 (2009), 1-38

por ciento de la población. En los años 2005 y 2006, la cobertura del SMI fue menor que en 2004, con un porcentaje de incidencia inferior al 1 por ciento. Utilizando la información procedente de la MCVL (quizá la más fiable) se puede afirmar que en el año 2004 había tan sólo 140.000 trabajadores del Régimen General de la Seguridad Social con empleos a tiempo completo sujetos al SMI en nuestro país, reduciéndose esa cifra a poco más de 100.000 personas en $2006 .^{11}$

\section{Estimación de los efectos del SMI sobre el empleo}

En esta sección se procederá a la estimación del efecto de los aumentos del SMI sobre el empleo. Para ello, se utilizarán dos bases de datos distintas. En primer lugar, los datos de la ECL permitirán analizar el impacto de los cambios en el índice de Kaitz sobre el empleo de las empresas españolas para el periodo 2000TI-2008TIV. En segundo lugar, se utilizarán los datos agregados de la EPA correspondientes al periodo 1981TI-2009TII para conocer el efecto de los cambios en el índice de Kaitz sobre las tasas de empleo de distintos colectivos. Previamente se procederá a resumir los resultados obtenidos en las principales investigaciones que han abordado esta cuestión.

La controversia teórica en relación con los efectos del salario mínimo sobre los niveles de empleo no se ha podido cerrar totalmente a partir de los datos existentes. No obstante, en el caso español parece que los efectos del SMI sobre el empleo no han sido especialmente negativos, manifestándose sólo en el colectivo de trabajadores más jóvenes y menos formados. En general, los trabajos de Pérez (1995), Dolado et al. (1996), Dolado y Felgueroso (1997), González (1997), Pérez et al. (2002), y González et al. (2003), han encontrado evidencia de un impacto significativo, pero débil, del SMI sobre el empleo adolescente (16 a 19 años). Los efectos sobre el resto de trabajadores son inapreciables. En estos artículos se estiman ecuaciones en las que la tasa de empleo de cada colectivo se explica en función del índice de Kaitz y de otras variables representativas de la evolución de la demanda y la oferta de trabajo, utilizando datos hasta finales de los años 90 procedentes de la EPA, la Contabilidad Nacional y la Encuesta de Salarios en Industria y Servicios. En concreto, las elasticidades estimadas de la tasa de empleo adolescente ante variaciones del índice de Kaitz oscilan entre -0,05 y -0,6 (véanse, respectivamente, González, 1997, y Pérez, 1995). En el caso de Dolado et al. (1996), el valor estimado es -0,15. Según este último resultado, se

\footnotetext{
11 Un estudio detallados sobre la incidencia del SMI en España a partir del análisis de diversas fuentes de información puede encontrarse en Cebrián et al. (2008).
} 
puede afirmar que una subida del 10 por ciento en el índice de Kaitz dará lugar a una disminución del empleo adolescente de sólo el 1,5 por ciento. Más recientemente, González et al. (2009) analizan los efectos del salario mínimo sobre el empleo del colectivo de trabajadores inmigrantes para el periodo 1996-2008, observando la existencia de un impacto negativo, en especial en el caso de las mujeres. Finalmente, Blázquez et al. (2009) no encuentran ninguna evidencia rotunda en cuanto al signo de estos efectos para el caso español a lo largo del periodo 2000-2008.

Los resultados de las investigaciones realizadas para otros países no son del todo concluyentes, si bien como señalan Neumark y Wascher (2007) en una reciente revisión de la literatura, dos tercios de los estudios analizados indican la existencia de efectos negativos, más o menos intensos, del salario mínimo sobre el empleo. Así, por ejemplo, es frecuente encontrar este tipo de efecto en el caso de los trabajadores adolescentes (Williams y Mills, 1998; Baker et al., 1999; Gindling y Terrel, 2007; Hyslop y Stillman, 2007). Por el contrario, otros autores no detectan ningún efecto negativo significativo (Card y Krueger, 1995; Manning y Machin, 1996; Machin y Manning, 1997; Lang y Kahn, 1998; Lemos, 2009). Incluso, en algunos casos (por ejemplo, Berstein y Schmitt, 1998) parecen existir evidencias de efectos positivos de los salarios mínimos sobre la distribución de la renta. No obstante, incidiendo en este último punto, otras investigaciones sostienen justo lo contrario. Por ejemplo, Neumark et al. (2006) encuentran que tales efectos son, en general, no significativos, y para algunos grupos de bajos ingresos, incluso negativos, en el caso de Brasil; coincidiendo también con la opinión de Bird y Manning (2008) para el caso de Indonesia. Por su parte, Addison et al. (2009) observan la existencia de efectos positivos sobre el empleo en algunos sectores, explicables a partir de las teorías del monopsonio y de los salarios de eficiencia. Finalmente, en un estudio reciente para el caso de los Estados Unidos, Bazen y Gallo (2009) establecen incluso una diferencia clara entre los efectos del salario mínimo federal y de los distintos salarios mínimos establecidos en cada estado, concluyendo que en el primer caso estos efectos sobre el empleo son negativos, mientras que en el segundo resultan positivos.

\subsection{Estimación de los efectos del aumento del SMI sobre el empleo con datos de la Encuesta de Coyuntura Laboral (ECL)}

En esta sección se utilizarán los datos de la ECL para el periodo 2000TI-2008TIV con el objetivo de analizar los efectos de los cambios en el SMI (medidos a través del índice de Kaitz) sobre los niveles de empleo de las empresas españolas. La amplitud del periodo de análisis, 2000-2008, 
permite estudiar con suficiente perspectiva el efecto de los cambios producidos a partir del año 2004 en la cuantía del SMI.

\subsubsection{Los datos}

La ECL es una encuesta a empresas realizada con periodicidad trimestral por el MTIN. En cuanto a sus características, la ECL incluye en su ámbito a todos los centros de trabajo con independencia de su tamaño (desde 1997 incorpora los centros de 1 a 5 trabajadores, antes excluidos) y utiliza como directorio el Fichero de Cuentas de Cotización a la Seguridad Social. La ECL aporta información sobre unas 12.000 empresas cada trimestre. Por otra parte, la ECL no incluye las actividades económicas de la agricultura y la Administración Pública, defensa y seguridad social.

Para analizar los efectos del SMI sobre el empleo es habitual utilizar una medida relativa del mismo. Esta medida es el índice de Kaitz (IK). En esta sección se utilizarán exactamente los mismos valores del índice de Kaitz que aparecen en el Gráfico 4 de la Sección 3. Se dispone así de 48 valores distintos de IK para cada año del periodo de análisis (años 2000 a 2008). A cada empresa de la muestra se le asigna un valor distinto del índice de Kaitz, dependiendo del sector al que pertenece y del trimestre correspondiente. Además de este indicador, en las estimaciones se introducirá un conjunto de variables explicativas que aparecen definidas en el Cuadro A.1 del Apéndice. Por su parte, el Cuadro A.2 contiene los estadísticos descriptivos de dichas variables (media y desviación típica) para las diferentes muestras utilizadas.

\subsubsection{El modelo}

Partiendo de las referencias disponibles en los trabajos previos, en esta investigación se analiza el efecto del SMI sobre el empleo de las empresas a partir de la estimación de la siguiente ecuación:

Logaritmo del número de trabajadores $=$ f(logaritmo del índice de Kaitz, coste laboral relativo de los trabajadores contratados al SMI, ciclo económico, características de las empresas, características de los trabajadores, comunidad autónoma, sector, variables temporales)

La variable dependiente es el logaritmo del número de 
trabajadores de la empresa (LOGTRAB) ${ }^{12}$. Esta variable se explica por un conjunto de factores entre los que destacan el logaritmo del índice de Kaitz y el coste laboral relativo de los trabajadores contratados al SMI. Además, se incluyen diversas variables que muestran la evolución del ciclo económico y las características de las empresas y de sus trabajadores. Finalmente, se introducen controles por comunidad autónoma, sector de actividad, año y trimestre. La estimación de la ecuación anterior por Mínimos Cuadrados Ordinarios (MCO) a partir de los datos de la ECL, permite identificar los múltiples efectos de todas esas variables sobre el empleo de cada empresa.

A continuación se mencionan las variables concretas que se incluyen dentro de cada grupo, indicando su efecto esperado sobre el empleo.

\section{Logaritmo del índice de Kaitz (LOGIK)}

El índice de Kaitz muestra la proporción que representa el SMI en relación al salario medio vigente en la rama de actividad a la que pertenece la empresa (calculados ambos para valores mensuales). Si realmente el empleo de las empresas es sensible a las elevaciones del SMI, el signo del coeficiente estimado para esta variable resultará negativo. (PSMIIK)

Coste laboral relativo de los trabajadores contratados al SMI

La información proporcionada por la ECL permite calcular el coste laboral relativo que el conjunto de trabajadores contratados al salario mínimo representa para la empresa. En primer lugar, la ECL reporta información sobre la proporción de trabajadores que cobran el SMI en relación a la plantilla total de la empresa. Denominaremos a esa variable PSMI:

$$
P S M I=\frac{\text { Número de trabajadores sujetos al SMI }}{\text { Número total de trabajadores }}
$$

Por otra parte, el índice de Kaitz se ha elaborado para doce ramas de actividad del siguiente modo:

\footnotetext{
12 Esta variable se introduce en logaritmos porque mejora la calidad de los resultados de la estimación, y además, facilita el cálculo de la elasticidad de la tasa de empleo ante cambios en el índice de Kaitz.
} 


$$
I K=\frac{S M I}{\text { Salario Medio }}
$$

El producto de las dos expresiones anteriores será PSMIIK:

$$
\text { PSMIIK }=\frac{\text { Número de trabajadores sujetos al SMI }}{\text { Número total de trabajadores }} * \frac{S M I}{\text { Salario Medio }}
$$

PSMIIK es, pues, una medida del coste que representa para la empresa la contratación de trabajadores al SMI en relación al hipotético coste laboral en que incurriría dicha empresa si todos sus trabajadores recibieran el salario medio correspondiente a la rama de actividad a la que dicha empresa pertenece. Es de esperar que las elevaciones en ese ratio den lugar a disminuciones en el empleo.

\section{Variables de ciclo}

La plantilla de las empresas responde no sólo a cambios en el SMI sino fundamentalmente a otros factores que tienen que ver, por ejemplo, con la evolución del ciclo económico. Para representar el efecto del ciclo se introducen en la ecuación la proporción de bajas (ceses, despidos...) sobre la plantilla a lo largo del trimestre (PBAJAS) y la proporción de altas (contrataciones) (PALTAS). También se introducen las previsiones del empresario sobre la evolución del empleo para el año y el trimestre siguientes (PREVEMA y PREVEMT). Se espera que la plantilla de la empresa crezca en las fases alcistas (cuando aumentan las contrataciones y se reducen los despidos, y cuando los empresarios prevén aumentos de plantilla para el año y el trimestre siguientes). De forma alternativa, también se utilizará como indicador del ciclo económico la proporción de vacantes en el trimestre sobre la plantilla total de la empresa (PVACAN).

\section{Características de las empresas}

Para captar la posible influencia de algunas características de las empresas sobre la evolución de su plantilla, se introduce en la ecuación un conjunto de variables que muestran si predomina o no la jornada partida (JORPAR), si la distribución de la jornada es irregular a lo largo del año (JORIRREG), así como el número de horas trabajadas (jornada anual 
pactada, JORPAC), y las horas extraordinarias realizadas por término medio por cada trabajador (HOREXT).

\section{Características de los trabajadores}

Para controlar las características de los trabajadores, en las estimaciones realizadas se incluyen las proporciones de mujeres (PMUJ), de trabajadores indefinidos (PIND), y de trabajadores a tiempo completo en la empresa (PTC).

\section{Comunidad Autónoma, sector, año y trimestre}

La ecuación incluye un conjunto de variables ficticias de comunidad autónoma para tener en cuenta los efectos diferenciales derivados de la localización geográfica de la empresa. También se incluyen conjuntos de variables ficticias para controlar el sector, el trimestre y el año.

\section{Las estimaciones}

El Cuadro 1 contiene la estimación de la ecuación de empleo. Hemos de señalar que aunque la ECL proporciona información para una misma empresa durante más de un trimestre, no es posible identificar a cada empresa para crear un panel de datos que permita seguirla. Por eso, todas las observaciones se tratan como si fueran independientes. La primera columna de la tabla muestra los coeficientes estimados y la segunda los valores del test-t. Cada coeficiente debe interpretarse como la medida del cambio en el número de trabajadores de la empresa (en logaritmos) inducido por un incremento unitario de la variable en cuestión, una vez tenidos en cuenta los efectos de todas las demás variables incluidas en la ecuación.

Comenzando con el análisis del efecto del índice de Kaitz sobre el empleo, la estimación muestra que esta variable es altamente significativa y tiene signo negativo. El valor de la elasticidad del empleo con respecto al índice de Kaitz puede calcularse mediante la expresión ( $\left.\mathrm{a}_{1}+\mathrm{a}_{2} \mathrm{PSMIIK}\right)$, donde $a_{1}$ es el coeficiente estimado para IK y a2 el de PSMIIK ${ }^{13}$. Para el

13 Obsérvese que al estar incluido el índice de Kaitz dentro de la variable PSMIIK, no es posible interpretar directamente el coeficiente de la variable IK como la elasticidad del empleo ante cambios en el índice de Kaitz (tanto el empleo como IK se miden en logaritmos). Si se definiera PSMIIK también en logaritmos, dicha elasticidad podría calcularse como la suma de los coeficientes de las dos variables, pero dado que las empresas que no tienen trabajadores contratados al SMI son mayoría en la muestra 
valor medio de PSMIIK, la elasticidad del empleo con respecto al índice de Kaitz es igual a -0,73. Por ello, es posible afirmar que un aumento del uno por ciento en la proporción que representa el salario mínimo en relación al salario medio de la economía da lugar a una reducción del 0,73 por ciento en el empleo de las empresas. Ha de tenerse en cuenta que el efecto estimado se refiere a un aumento del uno por ciento en IK, no en el SMI. En la práctica, los incrementos en el SMI van seguidos de aumentos mucho menores en IK, dado que simultáneamente también crece el salario medio de la economía.

\section{Cuadro 1 Estimación del efecto del índice de Kaitz sobre el empleo (MCO) (Datos: ECL, 2000TI-2008TIV). Variable dependiente: LOGTRAB.}

\begin{tabular}{lrr}
\hline & Coeficiente & Test- $t$ \\
\hline Constante & $1,698^{*}$ & 15,79 \\
LOGIK & $-0,724^{*}$ & $-5,19$ \\
PSMIIK & $-2,697^{*}$ & $-41,06$ \\
Variables de ciclo & & \\
PALTAS & $0,084^{*}$ & 11,62 \\
PBAJAS & $-0,070^{*}$ & $-16,70$ \\
PREVEMA & $0,092^{*}$ & 10,44 \\
PREVEMT & $0,274^{*}$ & 30,33 \\
Características de la empresa & & \\
JORPAR & $-1,157^{*}$ & $-197,91$ \\
JORIRREG & $0,479^{*}$ & 63,82 \\
JORPAC & $0,001^{*}$ & 121,34 \\
HOREXT & $0,035^{*}$ & 59,22 \\
Características de los trabajadores & & \\
PMUJ & $-0,103^{*}$ & $-8,99$ \\
PIND & $-0,349^{*}$ & $-35,04$ \\
PTC & $-0,013$ & $-0,82$ \\
Comunidad Autónoma & & \\
ANDALUCÍA & $-0,663^{*}$ & $-55,11$ \\
ASTURIAS & $-0,462^{*}$ & $-30,60$ \\
ARAGÓN & $-0,608^{*}$ & $-43,09$ \\
BALEARES & $-0,596^{*}$ & $-39,61$ \\
CANARIAS & $-0,546^{*}$ & $-37,47$ \\
CANTABRIA & $-0,657^{*}$ & $-44,01$ \\
\hline
\end{tabular}

(siendo en esos casos PSMI=0), al tomar logaritmos se perdería la mayor parte de las observaciones. 


\begin{tabular}{|c|c|c|}
\hline CASTILLA-LA MANCHA & $-0,548^{*}$ & $-41,66$ \\
\hline CASTILLA-LEÓN & $-0,691^{*}$ & $-50,80$ \\
\hline CATALUÑA & $-0,285^{*}$ & $-26,89$ \\
\hline COMUNIDAD VALENCIANA & $-0,576^{*}$ & $-47,25$ \\
\hline EXTREMADURA & $-0,854^{*}$ & $-58,73$ \\
\hline GALICIA & $-0,512^{*}$ & $-39,14$ \\
\hline MURCIA & $-0,529 *$ & $-34,14$ \\
\hline NAVARRA & $-0,655^{*}$ & $-43,50$ \\
\hline PAÍS VASCO & $-0,306^{*}$ & $-23,69$ \\
\hline LA RIOJA & $-0,915^{*}$ & $-65,03$ \\
\hline \multicolumn{3}{|l|}{ Sector } \\
\hline INDUSTRIA EXTRACTIVA & $0,215^{*}$ & 4,26 \\
\hline INDUSTRIA MANUFACTURERA & $0,233^{*}$ & 8,56 \\
\hline PRODUCCIÓN ENERGÍA ELÉCTRICA & $0,377^{*}$ & 4,18 \\
\hline CONSTRUCCIÓN & $-0,029 *$ & $-2,64$ \\
\hline HOSTELERÍA & $-0,358^{*}$ & $-15,54$ \\
\hline TRANSPORTE & $0,287^{*}$ & 7,38 \\
\hline INSTITUCIONES FINANCIERAS & $1,162^{*}$ & 12,27 \\
\hline ACTIVIDADES INMOBILIARIAS & $0,481^{*}$ & 19,12 \\
\hline EDUCACIÓN & $0,950 *$ & 27,62 \\
\hline SANIDAD & $1,141^{*}$ & 24,61 \\
\hline OTROS SERVICIOS & $-0,312^{*}$ & $-18,72$ \\
\hline \multicolumn{3}{|l|}{ Variables temporales } \\
\hline AÑO 2001 & 0,012 & 1,09 \\
\hline AÑO 2002 & 0,001 & 0,05 \\
\hline AÑO 2003 & 0,019 & 1,52 \\
\hline AÑO 2004 & $0,053^{*}$ & 4,91 \\
\hline AÑO 2005 & $0,109 *$ & 9,34 \\
\hline AÑO 2006 & $0,158^{*}$ & 12,35 \\
\hline AÑO 2007 & $0,198^{*}$ & 14,14 \\
\hline AÑO 2008 & $0,235^{*}$ & 17,18 \\
\hline TRIMESTRE II & $-0,006$ & $-0,81$ \\
\hline TRIMESTRE III & $-0,043^{*}$ & $-5,81$ \\
\hline TRIMESTRE IV & $-0,020^{*}$ & $-2,67$ \\
\hline $\mathrm{R}^{2}$ & \multicolumn{2}{|c|}{26,76} \\
\hline $\mathrm{F}(51,423.167)$ & \multicolumn{2}{|c|}{$3.049,63$} \\
\hline $\mathrm{N}^{\mathrm{o}}$ de observaciones & \multicolumn{2}{|c|}{423.219} \\
\hline
\end{tabular}

Categoría de referencia: Predomina la jornada continuada, no hay distribución irregular de la jornada, la empresa es de Madrid, del sector del comercio, el dato de referencia corresponde al primer trimestre, y el año de referencia es el 2000.

(*) Variable significativa al 5\%; (**) Variable significativa al 10\% 
En segundo lugar, se observa que el empleo de las empresas depende también de la importancia que el coste salarial de los trabajadores que reciben el SMI tiene en relación a la masa salarial global de la empresa. Esa variable, que en la ecuación recibe el nombre de coste laboral relativo de los trabajadores contratados al SMI, presenta una alta significatividad y tiene signo negativo. Es decir, el empleo de las empresas tiende a disminuir a medida que aumenta la proporción que representan los costes laborales derivados de la contratación de trabajadores sujetos al SMI en relación a los costes laborales que existirían si todos fuesen remunerados al salario medio. En otras palabras, parece que el impacto del SMI sobre el empleo es mayor cuanto más especializada esté la empresa en la contratación del tipo de trabajadores que reciben ese salario (muy jóvenes y muy poco cualificados).

De forma alternativa, se ha estimado el modelo incorporando la variable PSMI (porcentaje de trabajadores sujetos al SMI) en lugar del coste salarial de los trabajadores que reciben el SMI en relación a la masa salarial global de la empresa (PSMIIK), sin que los resultados referidos al impacto del índice de Kaitz se modifiquen. En este caso, el coeficiente del logaritmo de IK se puede interpretar directamente como la elasticidad del empleo ante cambios en dicho índice. El coeficiente estimado es significativo y su valor es 0,73 , que coincide con el calculado en la estimación principal. El resto de coeficientes de la ecuación apenas se modifican. ${ }^{14}$

En cuanto a las variables representativas del ciclo económico, obsérvese que, como era de esperar, el empleo de las empresas tiende a aumentar en los periodos de auge, cuando crece la proporción de nuevos contratados (altas) y disminuye la de ceses y despidos (bajas). El empleo crece también cuando las previsiones del empresario para el siguiente trimestre y el siguiente año son favorables (mejoran las expectativas).

En lo que se refiere a las características de las empresas, se observa que el empleo disminuye en los centros que tienen preferentemente jornada partida, mientras que aumenta cuando la distribución de la jornada es irregular y a medida que aumentan la jornada pactada y el número de horas extraordinarias por trabajador.

En cuanto al efecto de las características personales de los trabajadores, el empleo es mayor cuando la proporción de mujeres en la empresa es más reducida. También es mayor cuando disminuye la proporción de trabajadores con contrato indefinido. Por su parte, la proporción de trabajadores a tiempo completo no resulta significativa.

${ }^{14}$ Las estimaciones de este segundo modelo no se presentan en el artículo aunque se encuentran disponibles para su consulta. 
La ECL proporciona también información sobre el porcentaje de variación del empleo previsto por el empresario para el trimestre siguiente al actual. De este modo, es posible calcular el impacto de los cambios en el índice de Kaitz sobre la variación prevista en el empleo. En el Cuadro 2 se muestra dicha estimación. La variación prevista en el empleo (VARPTRAB) se explica por la variación en el índice de Kaitz (VARIK) y por un conjunto de factores que ya han sido considerados en la estimación anterior. La principal diferencia con el planteamiento previo es que ahora se utiliza la proporción de vacantes en el trimestre como indicador del ciclo. Los resultados de la estimación son peores que los obtenidos en el Cuadro 1. De hecho, el modelo tiene muy poca capacidad explicativa (el $\mathrm{R}^{2}$ es muy bajo), si bien la variable que representa la variación relativa en el índice de Kaitz es significativa y su coeficiente negativo. En definitiva, y a modo de conclusión de esta sección, parece que las estimaciones realizadas a partir de la información proporcionada por la ECL detectan la existencia de un efecto significativo y negativo del SMI, medido en términos relativos, sobre el empleo de las empresas españolas.

\begin{tabular}{|c|c|c|}
\hline \multicolumn{3}{|c|}{$\begin{array}{l}\text { Cuadro } 2 \text { Estimación del efecto del índice de Kaitz sobre el } \\
\text { empleo (MCO) (Datos: ECL, 2000TI-2008TIV). } \\
\text { dependiente: VARPTRAB }\end{array}$} \\
\hline & Coeficiente & Test- $t$ \\
\hline Constante & $0,014^{*}$ & 4,42 \\
\hline VARIK & $-0,028^{* *}$ & $-1,68$ \\
\hline PSMIIK & $-0,014$ & $-1,51$ \\
\hline \multicolumn{3}{|l|}{ Variables de ciclo } \\
\hline PVACAN & 0,012 & 1,62 \\
\hline \multicolumn{3}{|l|}{ Caracteristicas de la empresa } \\
\hline JORPAR & $0,003^{*}$ & 6,19 \\
\hline JORIRREG & $0,003^{*}$ & 4,00 \\
\hline JORPAC & $-0,000005^{*}$ & $-3,81$ \\
\hline HOREXT & $-0,0002^{*}$ & $-4,19$ \\
\hline \multicolumn{3}{|l|}{ Características de los trabajadores } \\
\hline PMUJ & $-0,0003$ & $-0,25$ \\
\hline PIND & $0,008^{*}$ & 6,78 \\
\hline PTC & $0,006^{*}$ & 3,57 \\
\hline \multicolumn{3}{|l|}{ Comunidad Autónoma } \\
\hline ANDALUCÍA & $-0,003^{*}$ & $-2,68$ \\
\hline ASTURIAS & $-0,005^{*}$ & $-4,18$ \\
\hline ARAGÓN & $-0,001$ & $-0,82$ \\
\hline BALEARES & $-0,020^{*}$ & $-10,77$ \\
\hline
\end{tabular}




\begin{tabular}{|c|c|c|}
\hline CANARIAS & $-0,007 *$ & $-5,92$ \\
\hline CANTABRIA & $-0,003^{* *}$ & $-1,67$ \\
\hline CASTILLA-LA MANCHA & $-0,002^{* *}$ & $-1,72$ \\
\hline CASTILLA-LEÓN & 0,001 & 0,53 \\
\hline CATALUÑA & $-0,004^{*}$ & $-5,43$ \\
\hline COMUNIDAD VALENCIANA & $-0,008^{*}$ & $-7,67$ \\
\hline EXTREMADURA & $-0,005^{*}$ & $-3,37$ \\
\hline GALICIA & $-0,002^{*}$ & $-1,99$ \\
\hline MURCIA & $-0,004^{*}$ & $-2,51$ \\
\hline NAVARRA & $-0,003^{*}$ & $-2,35$ \\
\hline PAÍS VASCO & $-0,005^{*}$ & $-4,72$ \\
\hline LA RIOJA & $-0,002$ & $-1,43$ \\
\hline Sector & & \\
\hline INDUSTRIA EXTRACTIVA & $-0,007 *$ & $-4,00$ \\
\hline INDUSTRIA MANUFACTURERA & $-0,002 *$ & $-3,13$ \\
\hline PRODUCCIÓN ENERGÍA ELÉCTRICA & $-0,004^{*}$ & $-2,48$ \\
\hline CONSTRUCCIÓN & $0,005^{*}$ & 4,45 \\
\hline HOSTELERÍA & 0,001 & 0,97 \\
\hline TRANSPORTE & 0,0004 & 0,34 \\
\hline INSTITUCIONES FINANCIERAS & $-0,003^{*}$ & $-3,37$ \\
\hline ACTIVIDADES INMOBILIARIAS & $0,008^{*}$ & 9,45 \\
\hline EDUCACIÓN & 0,0002 & 0,17 \\
\hline SANIDAD & $0,005^{*}$ & 4,69 \\
\hline Otros servicios & $0,003^{*}$ & 2,54 \\
\hline Variables temporales & & \\
\hline AÑO 2001 & $-0,003^{*}$ & $-2,80$ \\
\hline AÑO 2002 & $-0,003^{*}$ & $-3,05$ \\
\hline AÑO 2003 & $-0,003^{*}$ & $-2,97$ \\
\hline AÑO 2004 & $-0,002^{*}$ & $-2,09$ \\
\hline AÑO 2005 & $-0,002^{* *}$ & $-1,77$ \\
\hline AÑO 2006 & $0,002^{*}$ & 2,05 \\
\hline AÑO 2007 & $0,002^{* *}$ & 1,82 \\
\hline AÑO 2008 & $-0,016^{*}$ & $-13,68$ \\
\hline TRIMESTRE II & $-0,002^{*}$ & $-2,48$ \\
\hline TRIMESTRE III & $-0,014^{*}$ & $-18,11$ \\
\hline TRIMESTRE IV & $-0,003 *$ & $-3,28$ \\
\hline $\mathrm{R}^{2}$ & \multicolumn{2}{|c|}{0,48} \\
\hline $\mathrm{F}(48,411.443)$ & \multicolumn{2}{|c|}{29,98} \\
\hline $\mathrm{N}^{\mathrm{o}}$ de observaciones & \multicolumn{2}{|c|}{411.492} \\
\hline
\end{tabular}

Categoría de referencia: Predomina la jornada continuada, no hay distribución irregular de la jornada, la empresa es de Madrid, del sector del comercio, el dato de referencia corresponde al primer trimestre, y el año de referencia es el 2000. (*) Variable significativa al 5\%; (**) Variable significativa al 10\% 


\subsection{Estimaciones de los efectos del aumento del salario minimo sobre el empleo con datos agregados de la Encuesta de Población Activa (EPA)}

En esta sección se utilizará la información trimestral procedente de la Encuesta de Población Activa (EPA) y de la Encuesta Trimestral de Coste Laboral (ETCL) para analizar los efectos del índice de Kaitz sobre las tasas de empleo de distintos colectivos de población según edad y sexo. Las estimaciones se efectuarán para el periodo 1981TI-2009TII. Dado que la información sobre coste salarial anterior al año 1996 no distingue entre trabajadores a tiempo completo y a tiempo parcial, el SMI, el coste salarial ordinario medio y el índice de Kaitz han de calcularse por hora efectiva de trabajo. La utilización de magnitudes mensuales sólo sería posible si se dispusiera del coste salarial ordinario para los trabajadores a tiempo completo, ya que el SMI mensual viene dado para una jornada de ese tipo. Además, como ya se ha indicado, el índice de Kaitz se define en términos de salario neto.

Las estimaciones utilizan alternativamente como variable dependiente las tasas de empleo de la población adolescente (entre 16 y 19 años - TE1619), joven (entre 20 y 24 años - TE2024) y total (TE). Además, para cada tramo de edad se efectúan estimaciones conjuntas y por sexos. En total se dispone de 9 estimaciones. El objetivo perseguido consiste en calcular la elasticidad de la tasa de empleo de cada colectivo de población ante cambios en el índice de Kaitz (IKHORA). Además, en las estimaciones se controlan los efectos sobre la tasa de empleo del ciclo económico y de la distribución por edades y sectores de la población.

En cuanto al ciclo económico, sus efectos se recogen por medio de dos variables: la tasa de paro masculina correspondiente a los trabajadores de 25 a 54 años (TPM2554) y la tasa de actividad retrasada un periodo (TA-1). En lo que se refiere a la primera variable, hay consenso en considerar su carácter exógeno en este tipo de estimaciones. No obstante, cuando las estimaciones se realizan para la submuestra masculina es preferible reemplazar esta variable por la tasa de paro global entre 25 y 54 años (TP2554), al objeto de evitar una posible fuente de endogeneidad. Por su parte, la tasa de actividad sí podría ser considerada

endógena en una estimación de los determinantes de la tasa de empleo (por la propia definición de ambas). Por ese motivo, la tasa de actividad se instrumenta por su valor retrasado un periodo. Además, con ese mismo objetivo, en las estimaciones realizadas para la muestra conjunta (ambos sexos) se introduce en su lugar la tasa de actividad masculina retrasada 
un periodo (TAM-1). ${ }^{15}$ Se espera que la tasa de empleo de cada grupo de edad crezca conforme la economía mejora su nivel de actividad (esto es, a medida que cae TPM2554 y crece TA-1).

Para controlar los cambios producidos en la oferta de trabajo de cada colectivo, se introducirán en las ecuaciones las siguiente variables: el porcentaje de adolescentes de 16 a 19 años sobre el total de población (PO1619 para la estimación correspondiente al conjunto de población, POM1619 para la estimación correspondiente a los varones y POF1619 para las mujeres); y el porcentaje de jóvenes de 20 a 24 años (definiéndose también tres variables distintas, PO2024, POM2024 y POF2024). La evolución de las tasas de empleo de adolescentes y jóvenes podría verse influida por la mayor o menor disponibilidad de mano de obra en estos segmentos de población.

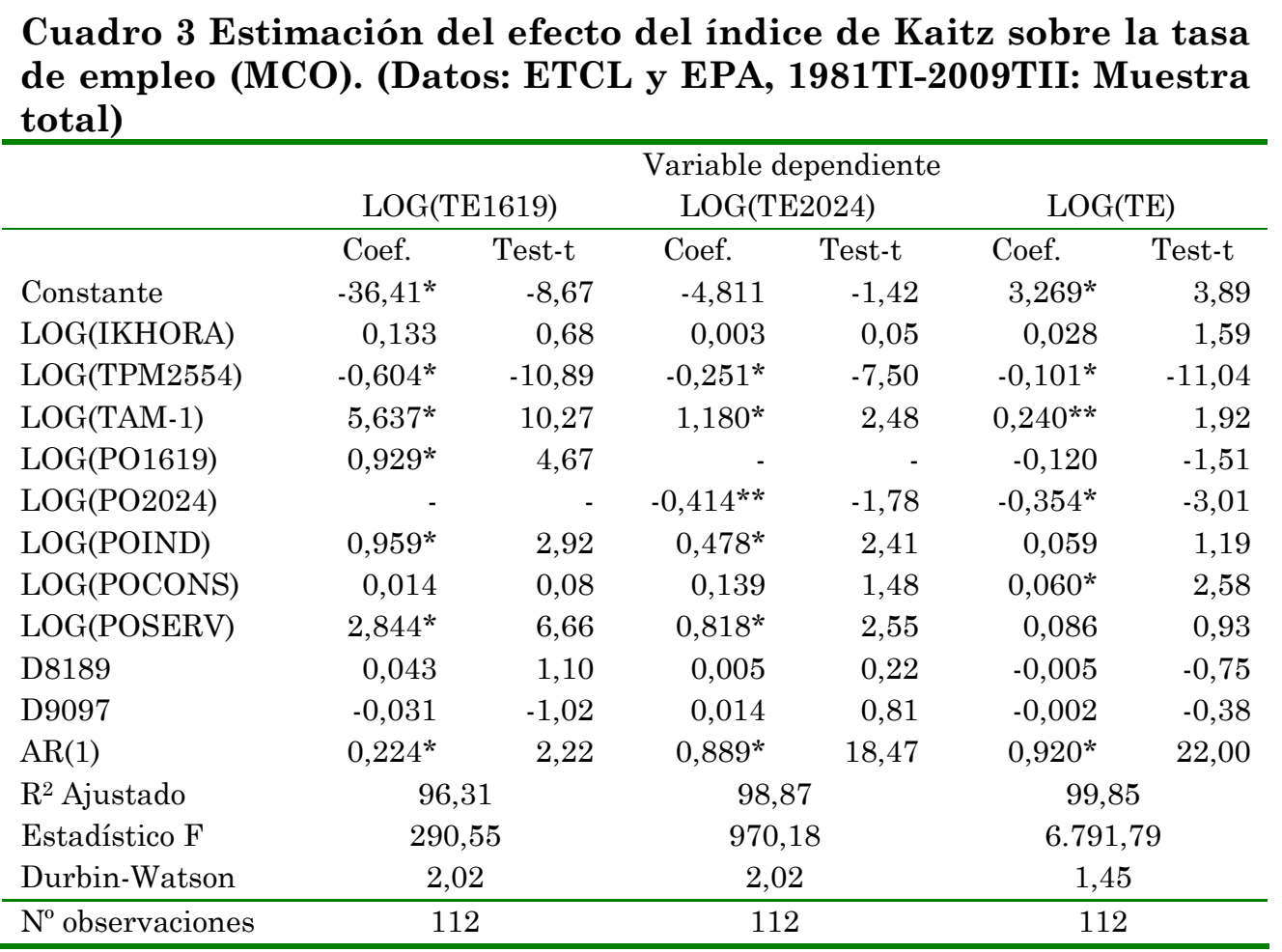

(*) Variable significativa al 5\%; (**) Variable significativa al 10\%

\footnotetext{
15 Para aproximar el efecto del ciclo económico se ha utilizado alternativamente la tasa de variación trimestral del PIB real. No obstante, esta variable no presenta buenos resultados en las estimaciones.
} 
Cuadro 4 Estimación del efecto del índice de Kaitz sobre la tasa de empleo (MCO). (Datos: ETCL y EPA, 1981TI-2009TII: Muestra masculina)

\begin{tabular}{|c|c|c|c|c|c|c|}
\hline & \multicolumn{6}{|c|}{ Variables dependientes } \\
\hline & \multicolumn{2}{|c|}{ LOG(TEM1619) } & \multicolumn{2}{|c|}{ LOG(TEM2024) } & \multicolumn{2}{|c|}{ LOG(TEM) } \\
\hline & Coef. & Test-t & Coef. & Test-t & Coef. & Test-t \\
\hline Constante & $-12,622^{*}$ & $-3,32$ & 0,380 & 0,12 & $-104,11$ & $-0,00$ \\
\hline LOG(IKHORA) & $-0,273$ & $-1,29$ & $-0,082$ & $-1,09$ & 0,003 & 0,15 \\
\hline LOG(TP2554) & $-0,845^{*}$ & $-9,26$ & $-0,233^{*}$ & $-5,50$ & $-0,098^{*}$ & $-8,27$ \\
\hline LOG(TA-1) & $1,974^{*}$ & 3,32 & $0,852^{* *}$ & 1,90 & 0,093 & 0,81 \\
\hline LOG(POM1619) & $0,767^{*}$ & 3,09 & - & - & $-0,345^{*}$ & $-2,04$ \\
\hline LOG(POM2024) & - & - & 0,061 & 0,21 & 0,007 & 0,02 \\
\hline LOG(POMIND) & $1,631^{*}$ & 3,68 & $0,513^{*}$ & 2,12 & $0,161^{*}$ & 2,63 \\
\hline LOG(POMCONS) & 0,033 & 0,17 & $0,305^{*}$ & 2,54 & $0,136^{*}$ & 4,55 \\
\hline LOG(POMSERV) & $1,068^{*}$ & 2,24 & $-0,392$ & $-0,95$ & 0,069 & 0,64 \\
\hline D8189 & 0,030 & 0,56 & $-0,006$ & $-0,24$ & $-0,009$ & $-1,31$ \\
\hline D9097 & $-0,053$ & $-1,36$ & 0,011 & 0,59 & $-0,004$ & $-0,81$ \\
\hline $\operatorname{AR}(1)$ & $0,430^{*}$ & 4,62 & $0,951^{*}$ & 30,84 & $0,999^{*}$ & 72,73 \\
\hline $\mathrm{R}^{2}$ Ajustado & 95,23 & & 98,54 & & 99 , & \\
\hline Estadístico F & 222,72 & & 750,49 & & 1.52 & \\
\hline Durbin-Watson & 2,05 & & 1,97 & & 1,6 & \\
\hline $\mathrm{N}^{0}$ observaciones & 112 & & 112 & & 11 & \\
\hline
\end{tabular}

Cuadro 5 Estimación del efecto del índice de Kaitz sobre la tasa de empleo (MCO). (Datos: ETCL y EPA, 1981TI-2009TII: Muestra femenina)

\begin{tabular}{|c|c|c|c|c|c|c|}
\hline & \multicolumn{6}{|c|}{ Variables dependientes } \\
\hline & \multicolumn{2}{|c|}{ LOG(TEF1619) } & \multicolumn{2}{|c|}{ LOG(TEF2024) } & \multicolumn{2}{|c|}{ LOG(TEF) } \\
\hline & Coef. & Test-t & Coef. & Test-t & Coef. & Test-t \\
\hline Constante & $-21,413^{*}$ & $-3,39$ & $-6,660^{*}$ & $-2,10$ & $4,003^{*}$ & 3,28 \\
\hline LOG(IKHORA) & 0,296 & 1,13 & 0,119 & 1,37 & 0,046 & 1,58 \\
\hline LOG(TPM2554) & $-0,571^{*}$ & $-7,93$ & $-0,234^{*}$ & $-7,51$ & $-0,092^{*}$ & $-7,22$ \\
\hline LOG(TA-1) & $5,516^{*}$ & 5,69 & $1,961^{*}$ & 3,71 & $0,584^{*}$ & 3,29 \\
\hline LOG(POF1619) & $1,846^{*}$ & 5,03 & - & - & $-0,243$ & $-1,03$ \\
\hline LOG(POF2024) & - & - & 0,197 & 0,62 & $-0,514^{* *}$ & $-1,88$ \\
\hline LOG(POFIND) & 0,065 & 0,22 & $-0,002$ & $-0,01$ & -0.029 & $-0,75$ \\
\hline LOG(POFCONS) & 0,027 & 0,42 & 0,023 & 0,99 & $0,017^{*}$ & 2,26 \\
\hline LOG(POFSERV) & $-0,317$ & $-0,43$ & 0,493 & 1,32 & $-0,247$ & $-1,51$ \\
\hline D8189 & 0,109 & 1,39 & 0,048 & 1,59 & 0,005 & 0,48 \\
\hline D9097 & 0,008 & 0,14 & 0,028 & 1,31 & 0,004 & 0,58 \\
\hline $\mathrm{AR}(1)$ & $0,635^{*}$ & 7,56 & $0,871^{*}$ & 17,99 & $0,988^{*}$ & 46,17 \\
\hline $\mathrm{R}^{2}$ Ajustado & 93,84 & & 98,59 & & 99 , & \\
\hline Estadístico F & 170,20 & & 776,22 & & 10.47 & \\
\hline Durbin-Watson & 2,06 & & 2,17 & & 1,8 & \\
\hline $\mathrm{N}^{0}$ observaciones & 112 & & 112 & & 11 & \\
\hline
\end{tabular}


Por su parte, el efecto que la composición sectorial del empleo puede tener sobre la tasa de empleo de cada colectivo se incorpora por medio de los porcentajes de empleo en la industria (POIND, POMIND y POFIND, dependiendo del sexo), la construcción (POCONS, POMCONS y POFCONS) y los servicios (POSERV, POMSERV y POFSERV), siendo la agricultura la categoría de referencia.

Finalmente, para recoger los posibles efectos que los cambios en la regulación del SMI han podido tener sobre la tasa de empleo, en las estimaciones se incluyen dos variables ficticias (D8189 y D9097) que identifican los periodos 1981-1989 (en el que había tres SMI distintos según la edad de los trabajadores) y 1990-1997 (dos SMI diferentes). Obsérvese que el periodo de referencia es 1998-2009, en el que el SMI es único.

Los Cuadros A.3 y A.4 del Apéndice contienen, respectivamente, las definiciones de las variables utilizadas y los estadísticos descriptivos de las mismas. Por su parte, los Cuadros 3 a 5 recogen los resultados de las estimaciones realizadas. En todas ellas las variables se expresan en logaritmos. Además, las variables han sido ajustadas por estacionalidad (utilizando el método de medias móviles) y se ha corregido la autocorrelación de primer orden a través de un esquema $\operatorname{AR}(1)$.

Los resultados de las estimaciones muestran que en ningún caso (ni siquiera para los adolescentes de 16 a 19 años) la tasa de empleo se ve afectada de forma significativa por los cambios en el índice de Kaitz. Este resultado no es extraño si se tiene en cuenta el bajo grado de cobertura del SMI en nuestro país.

Por otro lado, como era de esperar, la tasa de empleo de cualquier combinación de edad-sexo responde siempre de manera significativa y negativa a los aumentos de la tasa de paro entre 25 y 54 años (masculina o global, según casos), y de manera positiva (con una sola excepción) a los incrementos de la tasa de actividad retrasada (global o masculina, dependiendo de la estimación). El tamaño de los coeficientes estimados muestra también que esta respuesta al ciclo es, en general, más intensa a medida que disminuye la edad del colectivo considerado y en el caso de las mujeres (los trabajadores más jóvenes, especialmente si son mujeres, parecen estar más expuestos a los avatares de la coyuntura económica que el resto de población).

En cuanto al efecto de las variables que controlan las oscilaciones en la oferta de trabajo de cada colectivo (PO1619, PO2024...) su signo y significatividad varían considerablemente, por lo que no es posible encontrar un único patrón de conducta. Finalmente, en muchas de las estimaciones las tasas de empleo muestran también una sensibilidad a los cambios en la distribución sectorial del empleo. 
En definitiva, las estimaciones realizadas en esta sección no encuentran un efecto significativo de los cambios en el índice de Kaitz sobre las tasas de empleo de los adolescentes, de los jóvenes y del conjunto de la población.

\subsection{Evaluación del impacto sobre el empleo de los futuros aumentos en el SMI}

Las dos estimaciones anteriores difieren en cuanto a la evaluación del impacto sobre el empleo de los cambios en el índice de Kaitz. Así, mientras que utilizando los microdatos de la ECL se obtiene un efecto negativo de los aumentos de IK sobre el nivel de empleo de las empresas, las estimaciones con datos agregados de la EPA no confirman la existencia de efectos negativos de IK sobre las tasas de empleo de los diferentes colectivos de población. No obstante, esta discrepancia puede tener una explicación. Por una parte, las variables dependientes utilizadas en cada modelo son diferentes. Mientras que en el primer caso se trata del nivel de empleo de las empresas, en el segundo se trata de tasas de empleo para diferentes colectivos de población. Estas tasas de empleo se definen como el cociente entre el número de ocupados y la población en el intervalo de edad correspondiente (por ejemplo, entre 16 y 19 años en el caso de la tasa de empleo adolescente). En principio, es posible que los cambios en IK afecten al empleo pero no a la tasa de empleo, siempre que el denominador de dicha tasa también varíe en la misma proporción. En segundo lugar, los datos de las estimaciones realizadas a partir de la ECL se refieren al periodo 2000TI-2008TIV, mientras que en las estimaciones con datos de la EPA se utiliza una muestra más larga (1981TI-2009TII). En tercer lugar, el número de variables de control es mayor en el caso de las estimaciones realizadas a partir de la ECL. Al tratarse de microdatos, la información que proporciona la ECL es mucho más refinada y permite controlar mejor la variedad de factores que pueden incidir en el número de efectivos laborales de la empresa. Por último, en el caso de la ECL se introduce en las estimaciones el índice de Kaitz para doce ramas de actividad, mientras que en el caso de la EPA se emplea como variable independiente el índice de Kaitz general. En todo caso, la falta de significatividad de IK en las estimaciones que utilizan como variable dependiente la tasa de empleo es un resultado habitual en la literatura económica de nuestro país, excepto, como ya se ha indicado, en el caso del colectivo de 16 a 19 años.

En esta sección trataremos de evaluar el posible impacto de la propuesta del Gobierno de elevar el salario mínimo de 600 a 800 euros en el periodo 2008-2012. Para ello, utilizaremos el valor de la elasticidad del empleo con respecto a IK estimado a partir de la ECL. No se evaluará el 
impacto de la subida del SMI sobre la tasa de empleo de la economía, dado que en este caso no se han estimado efectos de IK significativamente distintos de cero.

Antes de proceder a este análisis, merece la pena precisar algunas cuestiones relevantes que tienen que ver directamente con el problema planteado. En primer lugar, hay que recordar que el SMI tiene muy poca incidencia en España (alrededor del 1 por ciento). En segundo lugar, el SMI es la referencia para la determinación del salario base de convenio correspondiente a la categoría profesional de inferior rango jerárquico. Se ha comprobado que, dependiendo del convenio, el salario base de esa categoría puede superar hasta en un 40 por ciento al SMI, siendo poco frecuente que esta diferencia sea menor del 10 por ciento ${ }^{16}$. Además, puede ocurrir que conforme aumenta el SMI, también lo haga el salario base de convenio de la categoría inferior, y con el objetivo de mantener el abanico salarial, los salarios base correspondientes al resto de categorías. Por consiguiente, dada la reducida cobertura del SMI, si sus aumentos tienen efectos negativos sobre el empleo ha de ser por su efecto inducido sobre la estructura de salarios pactados en los convenios colectivos. Éstos constituyen un segundo escalón de salario mínimos (por categoría, sector, provincia y empresa), a los que legalmente están sujetos la mayoría de los trabajadores españoles. Resulta difícil suponer que a medio plazo los aumentos del SMI no van a repercutir en incrementos semejantes de los salarios pactados en los niveles inferiores de la escala jerárquica y, por extensión, en el resto de salarios negociados.

En las estimaciones, se ha obtenido una elasticidad del empleo con respecto a IK igual a -0,73. Esto es, un aumento del 1 por ciento en IK da lugar a una reducción del empleo del 0,73 por ciento. Pero hay que tener en cuenta que un aumento del 1 por ciento en IK no supone que se haya producido un aumento del 1 por ciento en el SMI. Desde el año 2000 hasta el 2008 el SMI ha crecido un 41,24 por ciento, mientras que IK ha aumentado sólo un 6,45 por ciento. La razón de esta diferencia es que a medida que crece el SMI también lo hace el salario medio, y en muchas ocasiones con una intensidad mayor.

Cuadro 6 Estimación de la variación porcentual en el empleo derivada del aumento en el SMI

\begin{tabular}{cccc}
\hline Periodo & Variación \% SMI & Variación \% IK & Variación \% Empleo \\
\hline $2000-2008$ & 41,24 & 6,45 & $-4,70$ \\
$2009-2012$ & 33,33 & 5,21 & $-3,80$ \\
\hline
\end{tabular}

16 Véase, Cebrián et al. (2008). 
Dados los porcentajes de crecimiento observados para IK y para el SMI entre 2000 y 2008, y dada la elasticidad estimada del empleo ante cambios en IK, el Cuadro 6 muestra el impacto de tales cambios sobre el nivel de empleo de las empresas a lo largo de estos nueve años. También se muestra el impacto previsto de una subida de 600 a 800 euros en el SMI para el periodo 2009-2012. En estos cálculos se supone que la razón entre el crecimiento del SMI y del índice de Kaitz observada en el periodo anterior se mantiene en el futuro (aunque evidentemente la magnitud de la crisis económica puede alterar la conducta de los agentes y modificar esta relación). Obsérvese que en el periodo 2000-2008, dado el incremento relativo en IK y dado un valor de la elasticidad del empleo ante cambios en IK igual a -0,73, es posible afirmar que el empleo de las empresas disminuyó un 4,7 por ciento debido a la elevación del salario mínimo. Esta sería una valoración de la pérdida de empleo atribuible a las elevaciones del SMI correspondientes a los nueve años, suponiendo que todos los demás factores que influyen sobre el empleo no se hubieran modificado. Pero el empleo de las empresas se ve sometido a condicionantes cíclicos y de otro tipo que han contribuido a un incremento espectacular del mismo a lo largo del periodo considerado. Esto quiere decir que el reducido efecto negativo atribuible al SMI se ha diluido en el contexto de fuerte crecimiento económico experimentado por la economía española entre los años 2000 y 2008 (en otras palabras, ha sido perfectamente asimilable por la economía española). Por su parte, el Gobierno se ha planteado como objetivo para el periodo 2008-2012 la elevación del SMI de 600 a 800 euros, lo que supondría un incremento relativo del 33,33 por ciento en cuatro años. Si se mantuviera la relación estimada entre SMI e IK para el periodo anterior, ello daría lugar a un aumento del 5,21 por ciento en IK y, dada la elasticidad del empleo frente a IK, ello supondría finalmente una caída del empleo del 3,8 por ciento. No obstante, como ya se ha señalado, hay muchos otros factores (además del SMI) que pueden influir en el empleo de las empresas. El hecho de que esta reducción del empleo del 3,8 por ciento en cuatro años se manifieste o se diluya entre el crecimiento generado por otros factores (aumento de la productividad, mejora en el nivel de cualificación de los trabajadores...) dependerá básicamente de la evolución del ciclo económico. De hecho, en un contexto de crisis como el actual no parece que incrementos excesivos en el SMI puedan resultar beneficiosos para el empleo. Estos efectos negativos serían menores si en la negociación colectiva los salarios de las categorías más bajas (y por extensión los del resto de categorías) se elevaran en una proporción mucho menor que el SMI. Ello podría convertir al SMI al final del periodo en la referencia salarial exacta para las categorías laborales más bajas en muchos convenios colectivos. Por el contrario, si la distancia existente en 
Cebrián et al. / Revista de Economía Laboral 7 (2009), 1-38

la actualidad entre los salarios base de las categorías inferiores y el SMI se mantuviera en los términos actuales, entonces los efectos negativos sobre el empleo y la inflación serían apreciables.

\section{Conclusiones}

La literatura económica establece que la fijación de salarios mínimos por encima de los salarios de equilibrio puede generar desempleo. Ello es más probable para los trabajadores más jóvenes y menos cualificados, es decir, en el caso de los trabajadores de menor productividad. En este supuesto, si se produce una elevación del SMI por encima del crecimiento de la productividad de estos trabajadores no cualificados, es posible que las empresas lleven a cabo un proceso de sustitución de los mismos por trabajadores cualificados, incluso aunque el salario de estos últimos sea superior. Estos efectos dependen también del contexto económico.

Por otra parte, los datos que se han presentado en esta investigación han puesto de manifiesto el escaso grado de cobertura del SMI en nuestro país, lo que quizá explique por qué las estimaciones muestran que los efectos de los aumentos del SMI sobre el empleo son pequeños. De hecho, los efectos estimados de los cambios en el índice de Kaitz sobre las tasas de empleo no son significativamente distintos de cero, si bien parece existir un efecto significativo y negativo de IK sobre los niveles de empleo de las empresas. En todo caso, estos resultados son válidos para cambios pequeños del índice de Kaitz. Es más difícil precisar cómo se vería afectada dicha tasa de empleo en el supuesto de que el índice de Kaitz alcanzara el 60 por ciento del salario medio de la economía. Teniendo en cuenta que dicho índice se encuentra en la actualidad ligeramente por encima del 42 por ciento según la ETCL, ese objetivo supone un aumento de casi 18 puntos porcentuales, lo que implica un cambio que podría considerarse estructural. Indudablemente, la importancia del desafío requiere contemplar ese objetivo como un asunto de largo plazo. Téngase en cuenta, en este sentido, que aumentar el índice de Kaitz no es lo mismo que aumentar el SMI. En efecto, aunque el Gobierno está elevando considerablemente el SMI desde el año 2004, la repercusión de esos incrementos en el índice de Kaitz es mucho menor, debido a que a la vez que se eleva el SMI crecen también los salarios a lo largo de toda la distribución salarial.

Además, un elemento que debe ser tenido en cuenta a la hora de calibrar los posibles efectos negativos del SMI es el hecho de que la 
negociación colectiva establece en España un segundo escalón de salario mínimos, legalmente vinculantes, que, en general, están siempre por encima del SMI (incluso para las categorías laborales de menor cualificación). En este caso, el problema de elevar el SMI no se centra sólo en su repercusión sobre la tarifa salarial correspondiente a la categoría inferior, sino también en su efecto inducido sobre los salarios de categorías superiores que suelen guardar una cierta relación de proporcionalidad con las tarifas de convenio más bajas. Evidentemente, lo mejor para contener los costes laborales de las empresas (y con ello la inflación inducida y la consiguiente pérdida de poder adquisitivo de los salarios) sería que las elevaciones del SMI que inciden directamente en la categoría laboral inferior no se transmitieran de manera proporcional al resto de categorías. No obstante, debido a la rígida estructura salarial del mercado de trabajo español, por la que los salarios negociados cada año mantienen las diferencias existentes entre las distintas categorías profesionales, y a que en todo este proceso predomina el "efecto cascada", cualquier aumento del SMI se extiende más allá del grupo de trabajadores menos cualificados. La repercusión de estos ajustes indirectos y el efecto arrastre sobre los mínimos sectoriales puede hacer que la subida del SMI afecte no sólo a los salarios negociados más bajos, sino a todos aquellos que toman como referencia a aquél, terminando este proceso en un aumento del salario medio.

En todo caso, incluso si se consiguiera comprimir la distribución de los salarios por categorías, no debe olvidarse que el abaratamiento relativo del trabajo más cualificado puede conducir, como ya se ha señalado, a procesos de sustitución de mano de obra no cualificada por empleo cualificado.

En la investigación se han valorado los posibles efectos sobre el empleo derivados de una subida del salario mínimo de 600 a 800 euros al mes en el periodo 2008-2012, tal como figura en el programa del Gobierno. Esta iniciativa supone una subida del SMI del 33,33 por ciento en cuatro años. Dados los valores estimados de la elasticidad del empleo de las empresas ante cambios en IK, es posible afirmar que la subida del SMI hasta los 800 euros podría generar una reducción del empleo del 3,8 por ciento en el conjunto de los cuatro años, suponiendo que no cambian todos los demás factores que condicionan el empleo de las empresas. Esa reducción del empleo no es muy fuerte, y puede verse además compensada o, por el contrario, acentuada, por el efecto de otros factores (como el ciclo económico, los cambios en la productividad del trabajo, en la estructura de cualificaciones, etc.). Dado el ciclo negativo en que se encuentra la economía mundial y, por extensión, la española, 
es posible que un incremento del SMI tan importante acabe potenciando ligeramente el efecto destructor del empleo que ya tiene la propia crisis. En otras palabras, si bien en un contexto de crecimiento del empleo los pequeños efectos negativos que puede tener una elevación importante del SMI se diluyen en la dinámica positiva del mercado, en una época de crisis estos efectos van a reforzar el ciclo depresivo, tanto más cuanto más significativos sean los aumentos y cuanto más rápido se trasmitan los mismos a la estructura de salarios negociados en los convenios, especialmente a los de las categorías laborales más bajas.

Por otro lado, cumplir el objetivo de que el SMI represente el 60 por ciento del salario medio de la economía en un contexto como el actual también resulta complicado. Teniendo en cuenta lo sucedido estos últimos cuatro años, se puede concluir que elevar el índice de Kaitz de manera significativa a corto plazo exigiría subir el SMI en una magnitud muy elevada y en muy poco tiempo, de tal manera que el resto de los salarios no tuvieran tiempo de acomodarse. Sólo así podría conseguirse el resultado buscado a corto plazo. Ahora bien, la estructura salarial española probablemente terminaría por actualizar el resto de los salarios, de modo que el efecto de arrastre y los demás efectos indirectos absorberían buena parte del aumento del SMI. De este modo, a largo plazo se habría generado tan sólo un problema de inflación salarial que anularía el objetivo deseado. Por ello, el objetivo de que el SMI represente el 60 por ciento del salario medio de la economía debe tratar de alcanzarse a más largo plazo, si no se quiere que haya repercusiones significativas en el empleo, más si se tiene en cuenta el contexto de desaceleración en el que actualmente se encuentra la economía española.

\section{Bibliografía}

Addison, J. T., McKinley, L. B. y Cotti, Ch. D. (2009): "Do minimum wages rise employment? Evidence from the U.S. retail-trade sector", Labour Economics, 16, pp. 397-408.

Baker, M., Benjamin, D. y Stanger, S. (1999): "The highs and lows of the minimum wage effect: A time-series cross-section study of the Canadian Law”, Journal of Labor Economics, 17, pp. 318-350.

Bazen, S. y Le Gallo, J. (2009): "The state-federal dichotomy in the effects of minimum wages on teenage employment in the United States", Economic Letters, 105, pp. 267-269. 
Bernstein, J. y Schmitt, J. (1998): Making Work Pay: The Impact of the 1996-97 Minimum Wage Increase, Economic Policy Institute, Washington, D.C.

Bird, K. y Manning, C. (2008): "Minimum wages and poverty in a developing country: Simulations from Indonesia's Household Survey", World Development, 36, pp. 916-933.

Blázquez, M., Llorente, R. y Moral, J. (2009): Minimum wage and youth employment rates in Spain: New evidence for the period 2000-2008, Economic Analysis Working Paper Series, $N^{\circ} 2$, Univesidad Autónoma de Madrid.

Brown, Ch. Gilroy, C. y Cohen, A. (1982): "The effects of the minimum wage on employment and unemployment", Journal of Economic Literature, 20, pp. 487-528.

Card, D. y Krueger, A. B. (1995): Myth and measurement: The new economics of the minimum wages, Princeton University Press, Princeton.

Cebrián, I., Rodríguez, C., Toharia, L. y Pitarch, J. (2008): Estudio de los efectos de un aumento del salario mínimo interprofesional en el empleo, Informe elaborado para el Ministerio de Trabajo e Inmigración.

Dolado, J., Kramarz, F., Machin, S., Manning, A., Margolis, D. y Teulings, C. (1996): "The economic impact of minimum wages in Europe", Economic Policy, Octubre, pp. 219-372.

Dolado, J. J. y Felgueroso, F. (1997): "Los efectos del salario mínimo: evidencia empírica para el caso español”, Moneda y Crédito, 204, pp. 213254.

Georgiadis, A. (2008): Efficiency wages and the economic effects of the minimum wage: Evidence from a low-wage labour market, Discussion Paper $\mathrm{N}^{\mathrm{o}}$ 857, CEP, Londres.

Gindling, T. H. y Terrel, K. (2007): "The effects of multiple minimum wages throughout the labor market: The case of Costa Rica", Labour Economics, 14, pp. 485-511.

González, I. (1997): "Los efectos del salario mínimo sobre el empleo de adolescentes, jóvenes y mujeres: evidencia empírica para el caso español”, Cuadernos Económicos de ICE, 63, pp. 31-48.

González, I., Pérez, C. y Jiménez, S. (2003): "Los efectos del salario mínimo sobre el empleo juvenil en España: nueva evidencia con datos de Panel", Revista Asturiana de Economía, 27, pp. 147-168. 
González, I., Pérez, C. y Rodríguez, J. C. (2009): Los efectos del incremento en el Salario Mínimo Interprofesional sobre el empleo de los trabajadores inmigrantes, mimeo, Universidad de Valladolid.

Hyslop, D. y Stillman, S. (2007): "Youth minimum wage reform and the labour market in New Zealand", Labour Economics, 14, pp. 201-230.

Lang, K. y Kahn, S. (1998): "The effect of minimum wage laws on the distribution of employment: Theory and evidence", Journal of Public Economics, 69, pp. 67-82.

Lemos, S. (2009): "Minimum wage effects in a developing country", Labour Economics, 16, pp. 224-237.

Manning, A. (2003): Monopsony in motion: Imperfect competition in labor markets, Princeton University Press, Princeton.

Machin, S. y Manning, A. (1997): "Minimum wages and economic outcomes in Europe”, European Economic Review, 41, pp. 733-742.

Manning, A. y Machin, S. (1996): "Employment and the introduction of a minimum wage in Britain”, Economic Journal, 106, pp. 667-673.

Neumark, D., Cunningham, W. y Siga, L. (2006): "The effects of the minimum wage in Brazil on the distribution of family incomes: 19962001", Journal of Development Economics, 80, pp. 136-159.

Neumark, D. y Wascher, W. (2007): Minimum wages and employment, Discussion Paper N ${ }^{\circ}$ 2570, IZA, Bonn.

Pérez, C. (1995): Los efectos del salario mínimo sobre el empleo y el desempleo: evidencia empírica para España, Actas de la I Jornadas de Economía Laboral.

Pérez, C., González, I. y de Praga, M. D. (2002): "Los efectos simultáneos del salario mínimo sobre el empleo, la participación y la tasa de paro de los adolescentes españoles", Moneda y Crédito, 215, pp. 225-246.

Williams, N. y Mills, J. (1998): "Minimum wages effects by gender", Journal of Labor Research, 19, pp. 397-414. 


\section{Apéndice \\ Cuadro A.1 Definiciones de las variables procedentes de la ECL \\ Variable dependiente \\ LOGTRAB Logaritmo neperiano de los efectivos laborales de la empresa. En la terminología de la ECL, los efectivos laborales son los trabajadores por cuenta ajena por los que existe obligación de cotizar a la Seguridad Social en el último mes del trimestre de referencia}

VARPTRAB Variación relativa (en tantos por uno) de los efectivos laborales de la empresa prevista por el empresario para el trimestre siguiente al actual

Variables independientes

LOGIK Logaritmo neperiano del índice de Kaitz, que se define como el cociente entre el Salario Mínimo Interprofesional mensual neto y el coste salarial ordinario mensual neto para los trabajadores a tiempo completo. El índice se expresa en tantos por uno. Cada año se calcula el índice de Kaitz para doce ramas de actividad y para los cuatro trimestres. En cada trimestre, a cada empresa se le asigna el índice correspondiente a la rama de actividad a la que pertenece

VARIK Variación relativa (en tantos por uno) en el índice de Kaitz

PSMIIK Se obtiene multiplicando el índice de Kaitz (en tantos por uno) por la proporción de trabajadores sujetos al SMI en relación al empleo total de la empresa. Muestra la importancia relativa de los costes laborales derivados de la contratación de trabajadores al SMI con relación al coste laboral que existiría si todos los trabajadores fueran contratados al salario medio de la rama de Variables de ciclo

PALTAS Proporción (en tantos por uno) que representan las nuevas contrataciones de trabajadores producidas a lo largo del trimestre de referencia con respecto al total de efectivos laborales

PBAJAS Proporción (en tantos por uno) que representan las bajas de trabajadores producidas a lo largo del trimestre de referencia (por jubilación, finalización de contrato, baja voluntaria, despido...) con respecto al total de efectivos laborales

PREVEMA Variable dicotómica que toma el valor 1 si el empresario opina que la plantilla de la empresa va a crecer a lo largo del próximo año y 0 si opina que se mantendrá o disminuirá

PREVEMT Variable dicotómica que toma el valor 1 si el empresario opina que la plantilla de la empresa va a crecer a lo largo del próximo trimestre y 0 si opina que se mantendrá o disminuirá

PVACAN Proporción (en tantos por uno) que representan las vacantes producidas a lo largo del trimestre de referencia con respecto al total de efectivos laborales

\begin{tabular}{ll}
\hline Características de la empresa \\
\hline JORPAR & Variable dicotómica que toma el valor 1 si la mayor parte de los trabajadores de la empresa \\
& tienen jornada partida y 0 si tienen jornada continuada
\end{tabular}

JORIRREG Variable dicotómica que toma el valor 1 si en la empresa se ha establecido una distribución irregular de la jornada de trabajo a lo largo del año y 0 en caso contrario. Por distribución irregular de la jornada se entiende que la duración de la jornada diaria varíe por razones tales como sobrecarga de trabajo, acumulación de tareas, necesidades del mercado o, simplemente, porque en verano la jornada pasa de partida a continuada

JORPAC Número de horas anuales pactadas por trabajador a tiempo completo según el convenio colectivo vigente $o$, en su defecto, lo acordado con los trabajadores

HOREXT Número medio de horas extraordinarias realizadas en el trimestre por trabajador

Características de los trabajadores

PMUJ Proporción (en tantos por uno) de mujeres en la empresa con respecto al total de efectivos

PIND Proporción (en tantos por uno) de trabajadores que poseen contrato indefinido con respecto al total de efectivos laborales

PTC Proporción (en tantos por uno) de trabajadores que poseen contrato a tiempo completo con respecto al total de efectivos laborales 


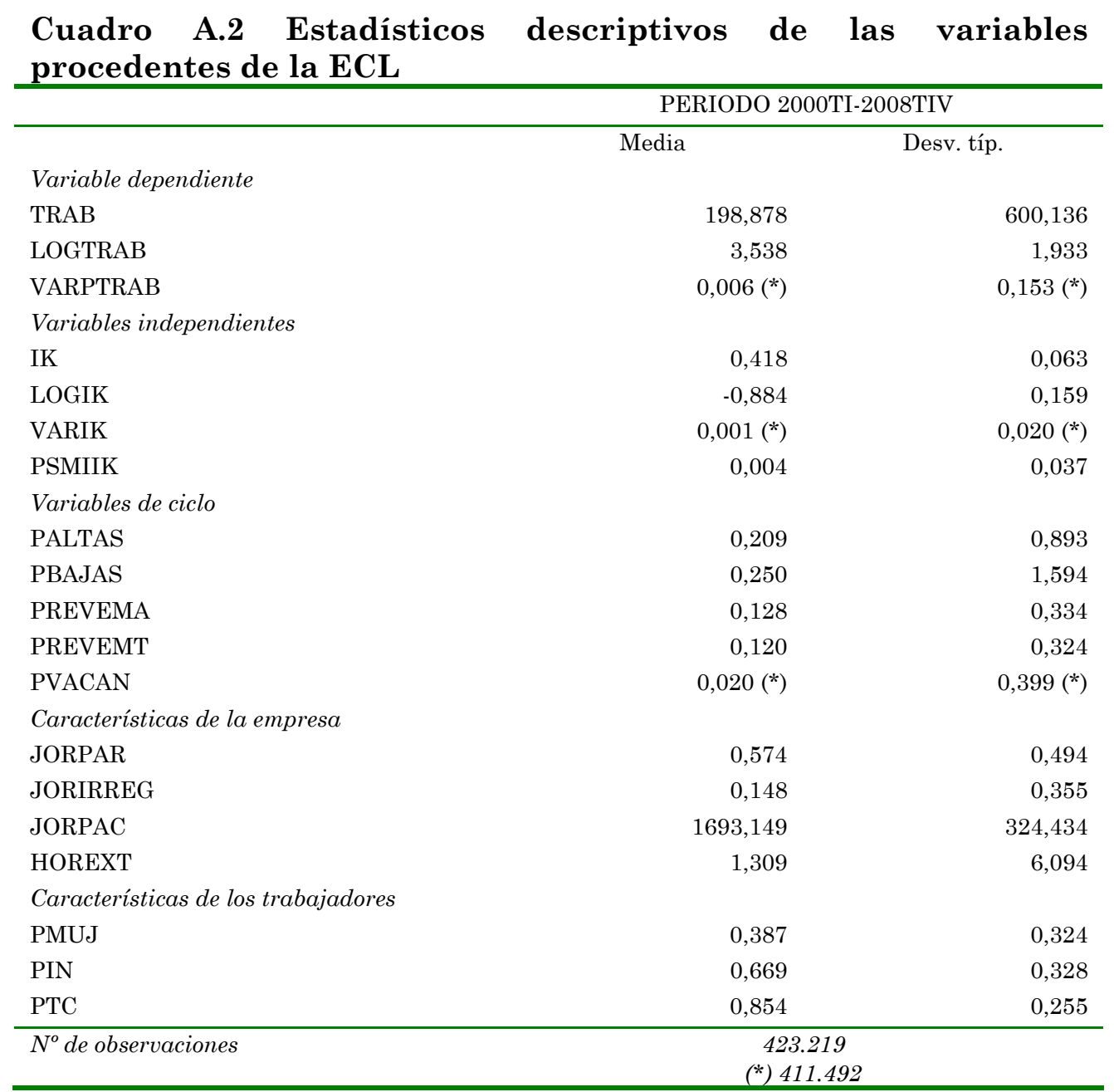

(*) En las estimaciones en las que la variable dependiente es la variación prevista en el empleo, el número de observaciones es menor, puesto que no se dispone de los valores de algunas variables para el primer trimestre de 2000. 


\section{Cuadro A.3 Definiciones de las variables procedentes de la EPA y la ETCL}

\begin{tabular}{|c|c|}
\hline \multicolumn{2}{|c|}{ Variables dependientes } \\
\hline TE1619 & Tasa de empleo de la población de 16 a 19 años, en porcentaje (EPA) \\
\hline TEM1619 & Tasa de empleo de la población masculina de 16 a 19 años, en porcentaje (EPA) \\
\hline TEF1619 & Tasa de empleo de la población femenina de 16 a 19 años, en porcentaje (EPA) \\
\hline TE2024 & Tasa de empleo de la población de 20 a 24 años, en porcentaje (EPA) \\
\hline TEM2024 & Tasa de empleo de la población masculina de 20 a 24 años, en porcentaje (EPA) \\
\hline TEF2024 & Tasa de empleo de la población femenina de 20 a 24 años, en porcentaje (EPA) \\
\hline $\mathrm{TE}$ & Tasa de empleo de la población total, en porcentaje (EPA) \\
\hline TEM & Tasa de empleo de la población masculina, en porcentaje (EPA) \\
\hline TEF & Tasa de empleo de la población femenina, en porcentaje (EPA) \\
\hline \multicolumn{2}{|c|}{ Variables independientes } \\
\hline IKHORA & $\begin{array}{l}\text { Índice de Kaitz definido como el cociente entre el Salario Mínimo } \\
\text { Interprofesional neto por hora efectiva de trabajo y el coste salarial ordinario } \\
\text { neto por hora efectiva para todo tipo de trabajadores (a tiempo completo y } \\
\text { parcial). El índice se expresa en tantos por ciento (ETCL) }\end{array}$ \\
\hline TP2554 & Tasa de paro de la población entre 25 y 54 años, en porcentaje (EPA) \\
\hline TPM2554 & Tasa de paro de la población masculina entre 25 y 54 años, en porcentaje (EPA) \\
\hline TA-1 & Tasa de actividad global retrasada un periodo, en porcentaje (EPA) \\
\hline TAM-1 & Tasa de actividad masculina retrasada un periodo, en porcentaje (EPA) \\
\hline PO1619 & $\begin{array}{l}\text { Porcentaje de adolescentes entre } 16 \text { y } 19 \text { años con relación al total de población } \\
\text { de } 16 \text { años o más (EPA) }\end{array}$ \\
\hline POM1619 & $\begin{array}{l}\text { Porcentaje de varones adolescentes entre } 16 \text { y } 19 \text { años con relación al total de } \\
\text { población de } 16 \text { años o más (EPA) }\end{array}$ \\
\hline POF1619 & $\begin{array}{l}\text { Porcentaje de mujeres adolescentes entre } 16 \text { y } 19 \text { años con relación al total de } \\
\text { población de } 16 \text { años o más (EPA) }\end{array}$ \\
\hline PO2024 & $\begin{array}{l}\text { Porcentaje de jóvenes entre } 20 \text { y } 24 \text { años con relación al total de población de } 16 \\
\text { años o más (EPA) }\end{array}$ \\
\hline POM2024 & $\begin{array}{l}\text { Porcentaje de varones jóvenes entre } 20 \text { y } 24 \text { años con relación al total de } \\
\text { población de } 16 \text { años o más (EPA) }\end{array}$ \\
\hline POF2024 & $\begin{array}{l}\text { Porcentaje de mujeres jóvenes entre } 20 \text { y } 24 \text { años con relación al total de } \\
\text { población de } 16 \text { años o más (EPA) }\end{array}$ \\
\hline POAGRI & Porcentaje de población ocupada en la agricultura (EPA) \\
\hline POMAGRI & Porcentaje de población masculina ocupada en la agricultura (EPA) \\
\hline POFAGRI & Porcentaje de población femenina ocupada en la agricultura (EPA) \\
\hline POIND & Porcentaje de población ocupada en la industria (EPA) \\
\hline POMIND & Porcentaje de población masculina ocupada en la industria (EPA) \\
\hline POFIND & Porcentaje de población femenina ocupada en la industria (EPA) \\
\hline POCONS & Porcentaje de población ocupada en la construcción (EPA) \\
\hline POMCONS & Porcentaje de población masculina ocupada en la construcción (EPA) \\
\hline POFCONS & Porcentaje de población femenina ocupada en la construcción (EPA) \\
\hline POSERV & Porcentaje de población ocupada en los servicios (EPA) \\
\hline POMSERV & Porcentaje de población masculina ocupada en los servicios (EPA) \\
\hline POFSERV & Porcentaje de población femenina ocupada en los servicios (EPA) \\
\hline D8189 & $\begin{array}{l}\text { Variable dicotómica que toma el valor } 1 \text { si el dato corresponde al periodo 1981- } \\
1989 \text { (en que había tres SMI distintos según edad) y } 0 \text { en caso contrario. }\end{array}$ \\
\hline D9097 & $\begin{array}{l}\text { Variable dicotómica que toma el valor } 1 \text { si el dato corresponde al periodo 1990- } \\
97 \text { (en que había dos SMI distintos según edad) y } 0 \text { en caso contrario. }\end{array}$ \\
\hline
\end{tabular}


Cuadro A.4 Estadísticos descriptivos de las variables procedentes de la EPA y la ETCL (Periodo 1981TI-2009TII)

\begin{tabular}{lrr}
\hline & Media & Desv. típ. \\
\hline TE1619 & 17,75 & 3,60 \\
TEM1619 & 21,40 & 4,19 \\
TEF1619 & 13,93 & 3,33 \\
TE2024 & 43,91 & 7,02 \\
TEM2024 & 49,90 & 7,48 \\
TEF2024 & 37,70 & 6,81 \\
TE & 44,02 & 4,93 \\
TEM & 59,25 & 3,50 \\
TEF & 29,67 & 7,23 \\
IKHORA & 43,06 & 6,20 \\
TP2554 & 13,46 & 3,89 \\
TPM2554 & 10,72 & 3,89 \\
TA-1 & 52,55 & 3,26 \\
TAM-1 & 68,33 & 2,38 \\
PO1619 & 7,48 & 1,65 \\
POM1619 & 7,88 & 1,75 \\
POF1619 & 7,10 & 1,55 \\
PO2024 & 9,83 & 1,22 \\
POM2024 & 10,30 & 1,29 \\
POF2024 & 9,38 & 1,16 \\
POAGRI & 10,10 & 4,67 \\
POMAGRI & 11,05 & 4,49 \\
POFAGRI & 8,28 & 4,72 \\
POIND & 21,25 & 3,16 \\
POMIND & 25,03 & 2,60 \\
POFIND & 14,03 & 2,88 \\
POCONS & 10,13 & 1,65 \\
POMCONS & 14,98 & 3,19 \\
POFCONS & 1,13 & 0,46 \\
POSERV & 58,48 & 6,39 \\
POMSERV & 48,91 & 4,31 \\
POFSERV & 76,52 & 7,13 \\
$N^{\circ}$ de observaciones & & \\
\hline & & 112 \\
\end{tabular}

